\title{
Heme oxygenase-1 deficiency accompanies neuropathogenesis of HIV-associated neurocognitive disorders
}

\author{
Alexander J. Gill, ${ }^{1}$ Colleen E. Kovacsics, ${ }^{1}$ Stephanie A. Cross, ${ }^{1}$ Patricia J. Vance, ${ }^{1}$ Lorraine L. Kolson, ${ }^{1}$ Kelly L. Jordan-Sciutto, ${ }^{2}$ \\ Benjamin B. Gelman, ${ }^{3}$ and Dennis L. Kolson ${ }^{1}$ \\ 'Department of Neurology, Perelman School of Medicine, University of Pennsylvania, Philadelphia, Pennsylvania, USA. ²Department of Pathology, School of Dental Medicine, University of Pennsylvania, \\ Philadelphia, Pennsylvania, USA. 르epartment of Pathology, University of Texas Medical Branch, Galveston, Texas, USA.
}

\begin{abstract}
Heme oxygenase-1 (HO-1) is an inducible, detoxifying enzyme that is critical for limiting oxidative stress, inflammation, and cellular injury within the CNS and other tissues. Here, we demonstrate a deficiency of HO-1 expression in the brains of HIVinfected individuals. This HO-1 deficiency correlated with cognitive dysfunction, HIV replication in the CNS, and neuroimmune activation. In vitro analysis of HO-1 expression in HIV-infected macrophages, a primary CNS HIV reservoir along with microglia, demonstrated a decrease in $\mathrm{HO}-1$ as HIV replication increased. HO-1 deficiency correlated with increased culture supernatant glutamate and neurotoxicity, suggesting a link among HIV infection, macrophage HO-1 deficiency, and neurodegeneration. HO-1 siRNA knockdown and HO enzymatic inhibition in HIV-infected macrophages increased supernatant glutamate and neurotoxicity. In contrast, increasing H0-1 expression through siRNA derepression or with nonselective pharmacologic inducers, including the CNS-penetrating drug dimethyl fumarate (DMF), decreased supernatant glutamate and neurotoxicity. Furthermore, IFN- $\gamma$, which is increased in CNS HIV infection, reduced HO-1 expression in cultured human astrocytes and macrophages. These findings indicate that $\mathrm{HO}-1$ is a protective host factor against HIV-mediated neurodegeneration and suggest that $\mathrm{HO}-1$ deficiency contributes to this degeneration. Furthermore, these results suggest that $\mathrm{HO}-1$ induction in the CNS of HIV-infected patients on antiretroviral therapy could potentially protect against neurodegeneration and associated cognitive dysfunction.
\end{abstract}

\section{Introduction}

Heme oxygenase-1 (HO-1) is a sentinel, detoxifying enzyme that is induced in response to numerous insults, including inflammation and oxidative stress (1-3). The potent antiinflammatory and cytoprotective actions of $\mathrm{HO}-1$ have identified it as a potential therapeutic target in CNS inflammatory and neurodegenerative diseases $(4,5)$. Among these diseases, HIV infection is associated with neurodegeneration that is thought to result from effects of persistent inflammation and oxidative stress in both systemic and CNS compartments that persist in individuals on antiretroviral therapy (ART) (6-9). Within the CNS, such effects are driven by HIV infection of macrophages and microglia and contribute to the continued prevalence of cognitive, motor, and behavioral deficits collectively known as HIV-associated neurocognitive disorders (HAND) $(10,11)$, which affect up to $50 \%$ of HIV-infected ART-treated individuals $(12,13)$. This high prevalence of HAND strongly underscores the need for adjunctive therapies that target the neuropatho-

Conflict of interest: Dennis Kolson has served as a paid consultant to Teva Neurosciences and Biogen-Idec Inc. and serves on the steering committee for the NIH/National Institute of Mental Health National NeuroAIDS Tissue Consortium. Benjamin Gelman has served as the chair of the National NeuroAIDS Tissue Consortium steering committee. Submitted: July 23, 2013; Accepted: August 4, 2014.

Reference information: J Clin Invest. 2014;124(10):4459-4472. doi:10.1172/JCI72279. logical processes associated with persistent inflammation and oxidative stress in HIV-infected individuals. We have identified HO-1 as a potential targetable modulator of HIV-associated neuropathological processes using human brain specimens from a large cohort of HIV-infected individuals and in vitro modeling of HIV-associated neurodegeneration.

Two heme oxygenase isoforms (HO-1 and HO-2) are expressed to varying levels in nearly all cell lineages, but only HO-1, the rapidly inducible isoform, is considered to be a critical mediator of the cellular response to injury (14). Elevated HO-1 expression has been observed in brain tissue from individuals with Alzheimer disease, Parkinson disease, and multiple sclerosis, perhaps reflecting a limited host-protective response against ongoing injury (15-17). The protective functions of HO-1 have been linked to the enzyme's degradation of heme, a strong prooxidant, and the subsequent generation of the antiinflammatory and antioxidative products, carbon monoxide, biliverdin, and bilirubin (14), although nonenzymatic cytoprotective functions of HO-1 through activation of oxidant-responsive transcription factors have also been proposed $(18,19)$. HO-1 catabolism of heme also releases free iron, which can contribute to cellular oxidative damage and toxicity. However, free iron rapidly induces expression of ferritin, a ubiquitous intracellular protein that binds and stores iron in a nontoxic form. Expression of HO- 1 is regulated through the NRF2-dependent antioxi- 
A
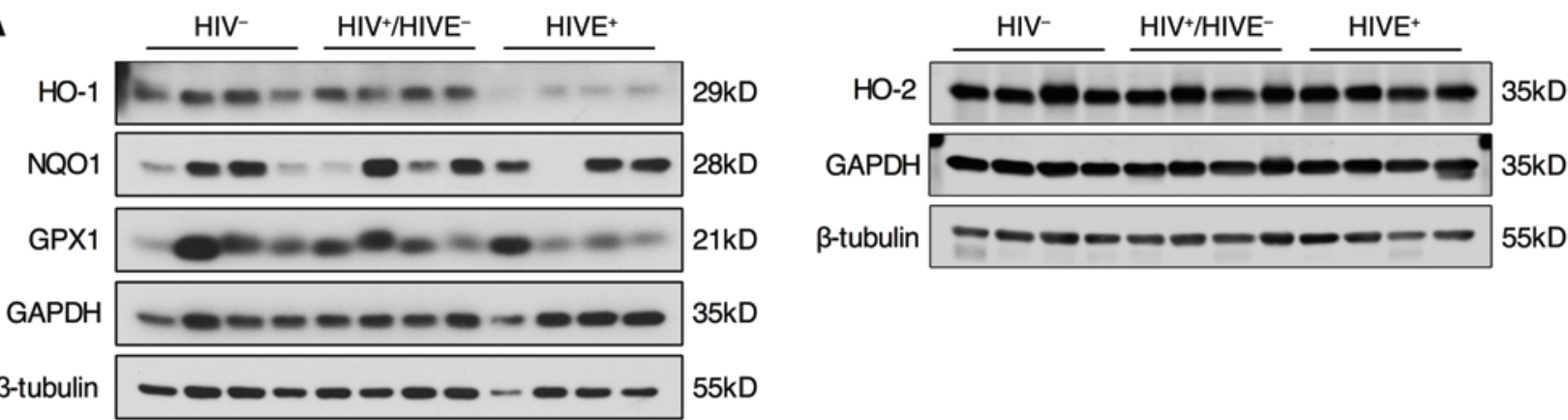

B

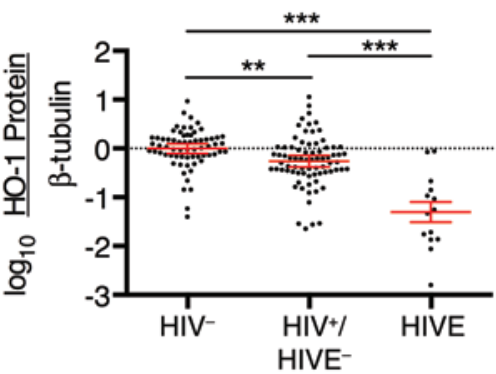

E

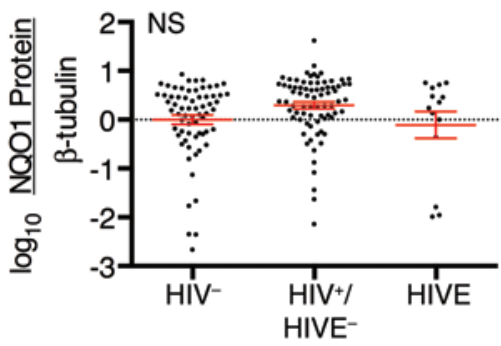

C

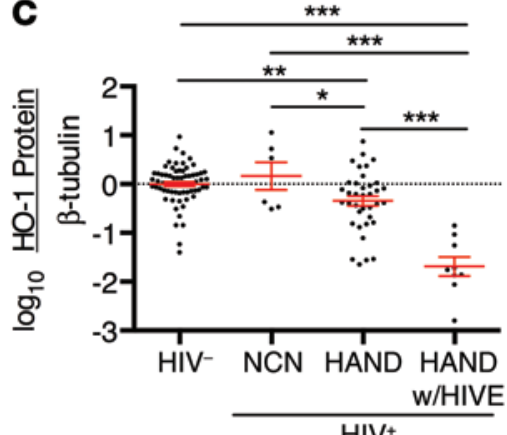

$\mathbf{F}$

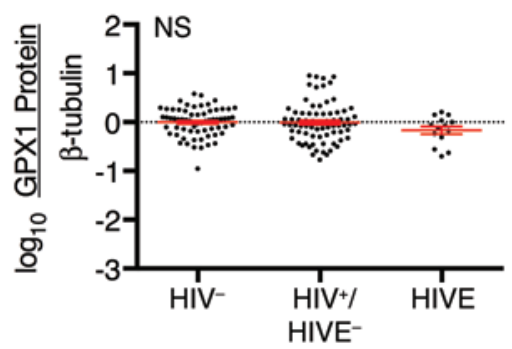

D

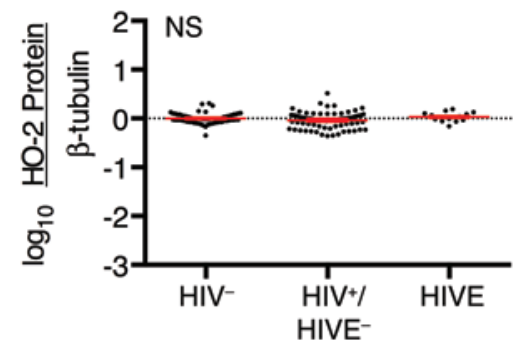

Figure 1. H0-1 expression is deficient in the DLPFC of HIV-infected subjects. Protein expression in the DLPFC was assessed by Western blot in 66 HIV-

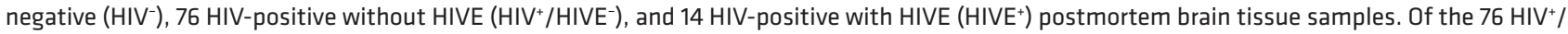

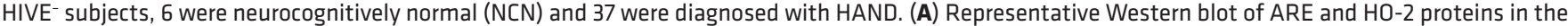
DLPFC of $\mathrm{HIV}^{-}, \mathrm{HIV}^{+} / \mathrm{HIVE}^{-}$, and $\mathrm{HIVE}^{+}$subjects. Protein expression levels were quantified by densitometry analysis, normalized to $\beta$-tubulin, and log transformed for comparison between groups for (B and C) HO-1, (D) HO-2, (E) NQ01, and (F) GPX1. The mean HIV- group protein expression was set to 0 (dotted line). Red lines indicate mean \pm SEM. Groups were analyzed by ANOVA with post hoc Holm-Sidak test. ${ }^{*} P<0.05 ;{ }^{* *} P<0.01 ;{ }^{* * *} P<0.001$.

dant response element (ARE), which regulates the induction of a coalition of antioxidant and detoxifying effector proteins (20).

Overexpression of HO-1 has been shown to be protective in animal models of inflammatory diseases, including cardiac ischemia (21), pulmonary hypoxia and inflammation (22, 23), and stroke (24); such studies have promoted the study of pharmacological HO-1 inducers in human diseases (25). Among these HO-1 inducers are the fumaric acid esters, notably dimethyl fumarate (DMF) and its primary in vivo metabolite monomethyl fumarate (MMF), which penetrates the CNS. Both DMF and MMF induce expression of ARE-driven genes, including HO-1, and modulate immune responses in various cell lineages through inhibition of $\mathrm{NF}-\kappa \mathrm{B}$ signaling, thereby promoting an antiinflammatory and antioxidative cellular state (reviewed in ref. 26). Recently, an oral DMF preparation, Tecfidera, received FDA approval for the treatment of multiple sclerosis, a disease characterized by recurrent neuroinflammation and oxidative stress.
Using our in vitro model of HIV-mediated neurodegeneration, in which HIV infection of monocyte-derived macrophages (HIV-MDM) induces release of soluble neurotoxins $(27,28)$, we have identified HO-1 as a key regulator of macrophage glutamate production and associated neurotoxicity. Through analysis of postmortem brain tissue specimens from 156 individuals, we demonstrated that HO-1 protein expression is deficient in the dorsolateral prefrontal cortex (DLPFC) of HIV-infected subjects and that this HO-1 deficiency is correlated with CNS viral load and markers of immune activation. Additionally, we have shown that HIV-associated HO-1 deficiency also occurs within the striatum, but not in the occipital cortex or cerebellum. We have further demonstrated that inducers of HO-1, including DMF and MMF, can ameliorate HIV-MDM glutamate release and neurotoxicity. These findings suggest that HO-1 deficiency contributes to HAND neuropathogenesis through modulation of neurotoxin production and that restoring CNS HO-1 expres- 


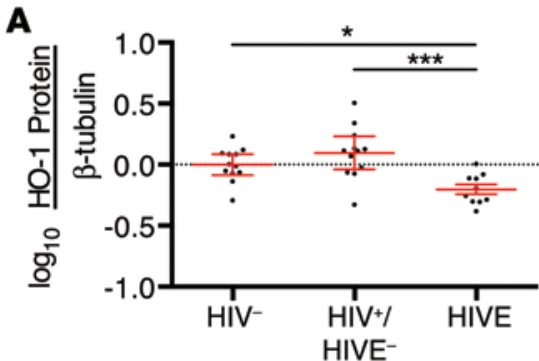

D

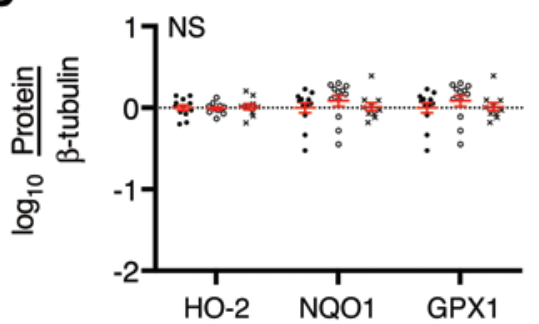

B

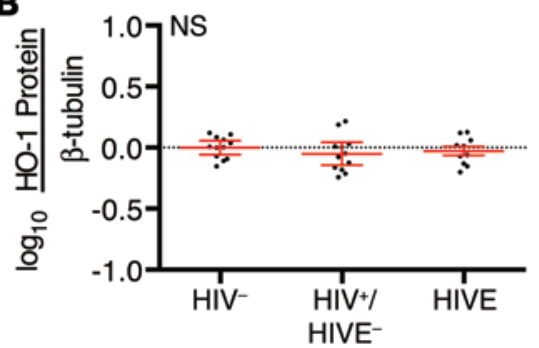

$\mathbf{E}$

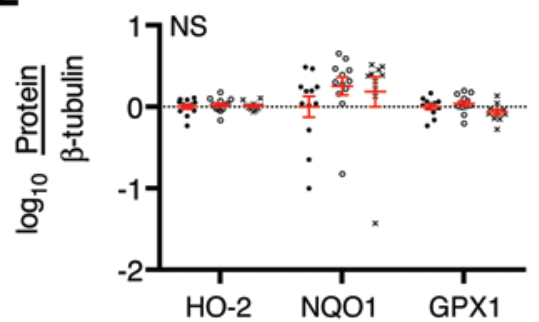

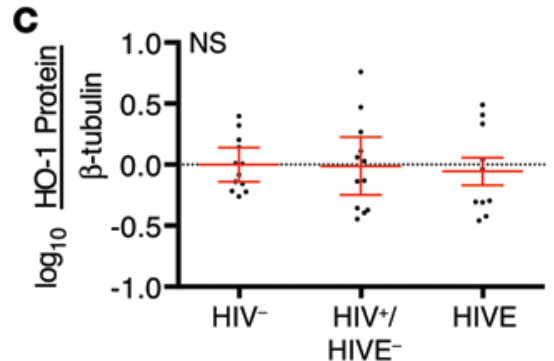

$\mathbf{F}$

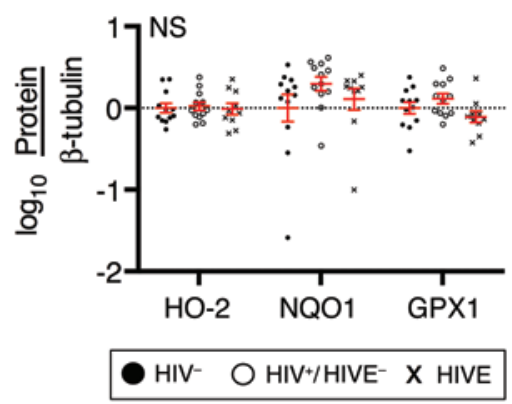

Figure 2. HO-1 is deficient in the striatum of HIV-infected subjects with HIVE. HO-1 protein expression as determined by Western blot in the (A) striatum (head of caudate), (B) occipital cortex, and (C) anterior cerebellum of $12 \mathrm{HIV}^{-}, 12 \mathrm{HIV}^{+} / \mathrm{HIVE}^{-}$, and $10 \mathrm{HIVE}$ subjects. In the same cohort, HO-2, NQ01, and GPX1 protein expression was determined by Western blot in the (D) striatum, (E) occipital cortex, and (F) cerebellum. Protein expression levels were quantified by densitometry analysis, normalized to $\beta$-tubulin, and log transformed for comparison between groups. The mean $\mathrm{HIV}^{-}$group protein expression was set to 0 (dotted line). Red lines indicate mean \pm SEM. Groups were analyzed by ANOVA with post hoc Holm-Sidak test. ${ }^{*} P<0.05$; ${ }^{* * *} P<0.001$.

sion could attenuate neurodegenerative processes and thereby reduce the persistent risk of HAND in ART-treated individuals.

\section{Results}

HO-1 protein expression is reduced in the prefrontal cortex of HIVinfected subjects. To determine the expression of HO- 1 in the brains of HIV-infected individuals, we used Western blotting to analyze the expression of HO-1, other canonical ARE effector proteins, and HO-2 in fresh-frozen autopsy specimens of DLPFC tissue from subjects enrolled in a previously described National NeuroAIDS Tissue Consortium (NNTC) cohort (29). These included samples from HIV-negative subjects ( $\left.\mathrm{HIV}^{-}, n=66\right)$, and HIV-positive subjects with and without HIV encephalitis (HIVE) (HIVE ${ }^{+}$ $n=14 ; \mathrm{HIV}^{+} / \mathrm{HIVE}^{-}, n=76$, respectively). There was a significant reduction in HO-1 protein expression in the DLPFC of $\mathrm{HIV}^{+}$ HIVE- $^{-}$individuals in comparison with $\mathrm{HIV}^{-}$controls, with the $\mathrm{HIVE}^{+}$subgroup showing even greater HO-1 deficiency (Figure 1, A and B, and Supplemental Figure 1A; supplemental material available online with this article; doi:10.1172/JCI72279DS1). HO-1 expression did not correlate significantly with age or postmortem autopsy interval within the full cohort or within the $\mathrm{HIV}^{-}, \mathrm{HIV}^{+}$/ $\mathrm{HIVE}^{-}$, or $\mathrm{HIVE}^{+}$subgroups (data not shown).

Further subgroup analysis revealed that HO-1 expression was reduced in $\mathrm{HIV}^{+} / \mathrm{HIVE}^{-}$subjects diagnosed with HAND compared with those diagnosed as neurocognitively normal when normalized to $\beta$-tubulin (Figure 1C), although this comparison was not significant when GAPDH expression was used to normalize (Supplemental Figure 1B). Notably, the $2 \mathrm{HIVE}^{+}$subjects who did not demonstrate HO-1 deficiency (Figure 1B and Supplemental Figure $1 \mathrm{~A}$ ) had neurocognitive impairment attributed to causes other than HAND. Use of ART was not associated with a significant dif- ference in $\mathrm{HO}-1$ expression levels in either $\mathrm{HIV}^{+} / \mathrm{HIVE}^{-}$or $\mathrm{HIVE}^{+}$ subjects (Supplemental Figure 2).

There were no significant differences in expression of either HO-2, a ubiquitously expressed heme oxygenase isoform (47\% homology with HO-1) that is not regulated through the ARE, or the ARE effector protein NADPH quinone oxidoreductase 1 (NQO1) among the $\mathrm{HIV}^{-}, \mathrm{HIV}^{+} / \mathrm{HIVE}^{-}$, and $\mathrm{HIVE}^{+}$subgroups (Figure 1, D and E, and Supplemental Figure 1, C and D). Similarly, expression of the ARE effector protein glutathione peroxidase 1 (GPX1) did not significantly differ among these groups when normalized to $\beta$-tubulin (Figure 1F), although GPX1 expression showed a significant decrease in $\mathrm{HIVE}^{+}$subjects compared with $\mathrm{HIV}^{+} / \mathrm{HIVE}^{-}$and $\mathrm{HIV}^{-}$subjects when normalized to GAPDH (Supplemental Figure 1E). We attempted to analyze NRF2 (the major ARE transcriptional regulator) by Western blot in the DLPFC. Using an extended panel of commercially available NRF2 antibodies, we were unable to reliably detect immunoreactivity consistent with NRF2 protein expression, despite detecting transfected $N R F 2$ gene products in HEK cells (Supplemental Figure 3). We therefore cannot confirm NRF2 protein expression levels in brain tissue; however, the lack of significant differences in the expression of the ARE proteins NQO1 and GPX1 in the DLPFC suggests that NRF2-dependent ARE activity is not altered by HIV-infection.

Brain HO-1 protein deficiency in HIV infection varies regionally. To determine whether brain HO-1 deficiency in HIV infection is restricted to the DLPFC, we similarly analyzed HO- 1 protein expression in the striatum (head of the caudate), occipital cortex, and cerebellum (anterior cerebellar cortex) in $12 \mathrm{HIV}^{-}, 12$ $\mathrm{HIV}^{+} / \mathrm{HIVE}^{-}$, and 10 HIVE cases. This regional analysis cohort was matched for age, post mortem interval, sex, race, and ethnicity. We observed a significant reduction in $\mathrm{HO}-1$ protein expres- 

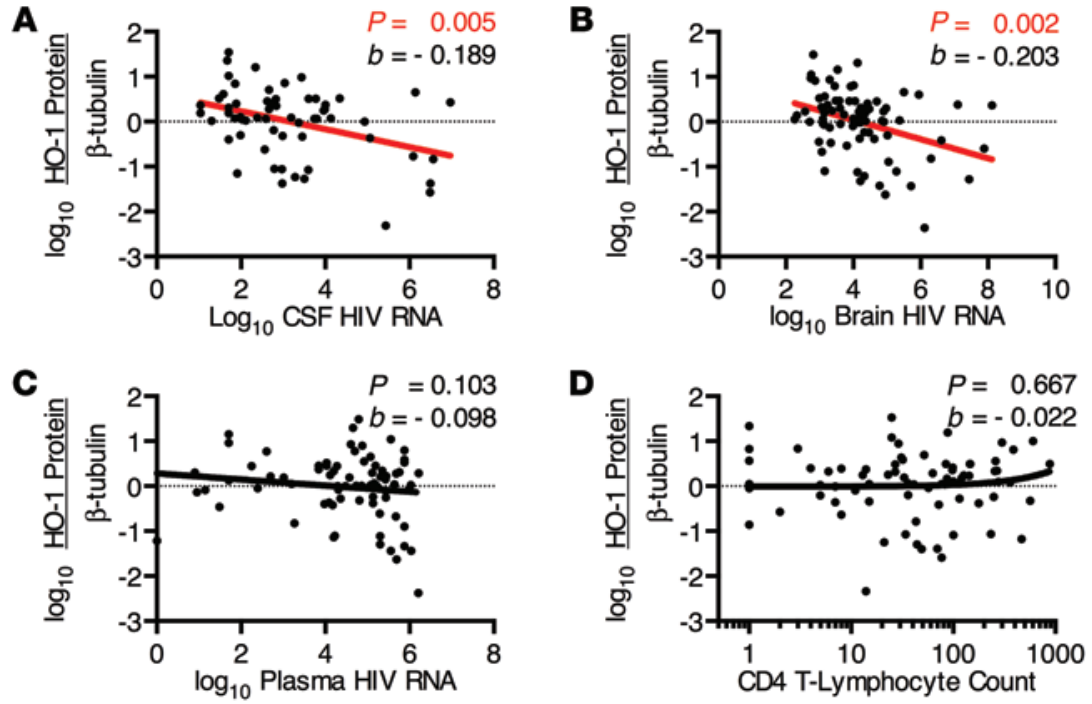

Figure 3. DLPFC HO-1 protein expression correlates with CSF and brain HIV RNA levels. Samples were derived from the $\mathrm{HIV}^{+}$cohort for all subjects with detectable viral loads. Correlations were determined between DLPFC HO-1 protein expression, as determined by Western blot and densitometry analysis normalized to $\beta$-tubulin, and HIV RNA in (A) CSF, (B) brain parenchyma, and (C) plasma, and (D) plasma CD4 T lymphocyte count in $\mathrm{HIV}^{+}$subjects. Associations were determined by multivariate linear regression with $\alpha=0.01$. Red regression lines denote significant trends. sion in the striatum of $\mathrm{HIVE}^{+}$subjects compared with $\mathrm{HIV}^{-}$and $\mathrm{HIV}^{+} / \mathrm{HIVE}^{-}$subjects (Figure 2A and Supplemental Figure 4A). However, we did not find any significant changes in $\mathrm{HO}-1$ protein expression in the occipital cortex or cerebellum (Figure 2, B and C, and Supplemental Figure 4, B and C). In each of these regions, the expression of HO-2, NQO1, and GPX1 proteins in the HIV group did not significantly differ from that in either the $\mathrm{HIV}^{+}$/ $\mathrm{HIVE}^{-}$or $\mathrm{HIVE}^{+}$groups. (Figure 2, D-F, and Supplemental Figure $4, \mathrm{D}-\mathrm{F})$, a finding consistent with that in the DLPFC.

HO-1 prefrontal cortex deficiency correlates with CNS viral load, type I IFN response, and macrophage activation. We further sought to determine the relationships among HIV replication and associated markers of immune activation and $\mathrm{HO}-1$ expression. In $\mathrm{HIV}^{+}$ subjects, prefrontal cortex HO-1 expression correlated negatively with both cerebrospinal fluid (CSF) and prefrontal cortex parenchyma HIV RNA load, but not with plasma viral load or CD4 ${ }^{+}$ $\mathrm{T}$ lymphocyte counts (Figure 3, A-D, and Supplemental Figure $5, \mathrm{~A}-\mathrm{D})$. This suggests that CNS HO-1 deficiency associates with ongoing HIV replication within the CNS compartment, but not with HIV replication in the periphery. As increased levels of markers of immune activation and the inflammatory type I IFN pathway are associated with the presence of HAND and high levels of brain parenchyma HIV RNA $(30,31)$, we assessed the mRNA expression of several markers of immune activation within our $\mathrm{HIV}^{+}$cohort. Expression of mRNA of the type I IFN-inducible genes ISG15 and MX1 correlated negatively with $\mathrm{HO}-1$ expression (Figure 4, A and B, and Supplemental Figure 6, A and B), while no significant correlation with mRNA expression of $I R F 1$, a predominantly type II IFN response gene (32), was observed (Figure 4C and Supplemental Figure 6C). Expression of mRNA of the macrophage/microglia activation marker and haptoglobin-hemoglobin scavenging receptor CD163 (33) correlated negatively with HO-1 expression (Figure 4D and Supplemental Figure 6D). The link to CD163, which is expressed by perivascular macrophages as well as a unique subset of ramified gray matter microglia in HIVE $(34,35)$, was relatively specific, as the macrophage activation marker CD68 and the cytotoxic T lymphocyte marker CD8A were not correlated significantly with $\mathrm{HO}-1$ (Figure 4, E and F, and
Supplemental Figure 6, E and F). These results suggest that prefrontal cortex HO-1 deficiency in HIV-infected individuals may be induced by CNS HIV replication and/or the associated innate immune activation of macrophages/microglia.

HO-1 protein deficiency in HIV-infected macrophages occurs concomitantly with increased supernatant glutamate and neurotoxicity. Many published studies, including our own, have linked HIV-associated macrophage/microglial immune activation to neuronal injury and neurodegeneration in vitro and in vivo $(27,36-40)$. We therefore investigated the link among macrophage HIV infection, HO-1 expression, and associated neuronal injury in our in vitro HIV neurodegeneration model system. Analogous with the brain HO-1 deficiency that we observed in $\mathrm{HIV}^{+}$individuals, HIV infection of MDM in vitro drastically reduced HO-1 expression. This reduction in HIV-MDM HO-1 expression was progressive and time dependent, typically showing a significant drop within 6 to 9 days after virus inoculation as replication approached its highest level (Figure 5, A and B). Furthermore, in contrast with this progressive HO-1 reduction, the expression of other ARE proteins (superoxide dismutase 1 [SOD1]; ferritin heavy chain 1 [FTH1]; peroxiredoxin 1[PRDX1]; glutathione S-transferase pi 1 [GSTP1]; thioredoxin reductase 1 [TRXR1]; NQO1; GPX1) was not consistently altered during infection (Figure 5C). Several of these proteins showed a transient increase from basal levels immediately after infection (day 0), but only HO-1 exhibited a progressive and persistent change from baseline over the course of infection. This suggests that the decrease in $\mathrm{HO}-1$ protein expression in HIV-MDM is relatively specific and not a consequence of global suppression of ARE-driven gene expression. Finally, we did not observe a decrease in expression of HO-2, which further suggests that the decrease of the HO- 1 isoform is relatively specific.

Associated with this selective HO-1 loss in HIV-MDM was a concomitant increase in extracellular levels of glutamate (Figure 5D), which is released from HIV-infected macrophages and microglia $(41,42)$. Notably, glutamate has been shown to be elevated in the CSF of HIV-infected individuals and CSF glutamate levels are correlated with the severity of HAND and brain atrophy (43). As expected, increased HIV-MDM supernatant glutamate 

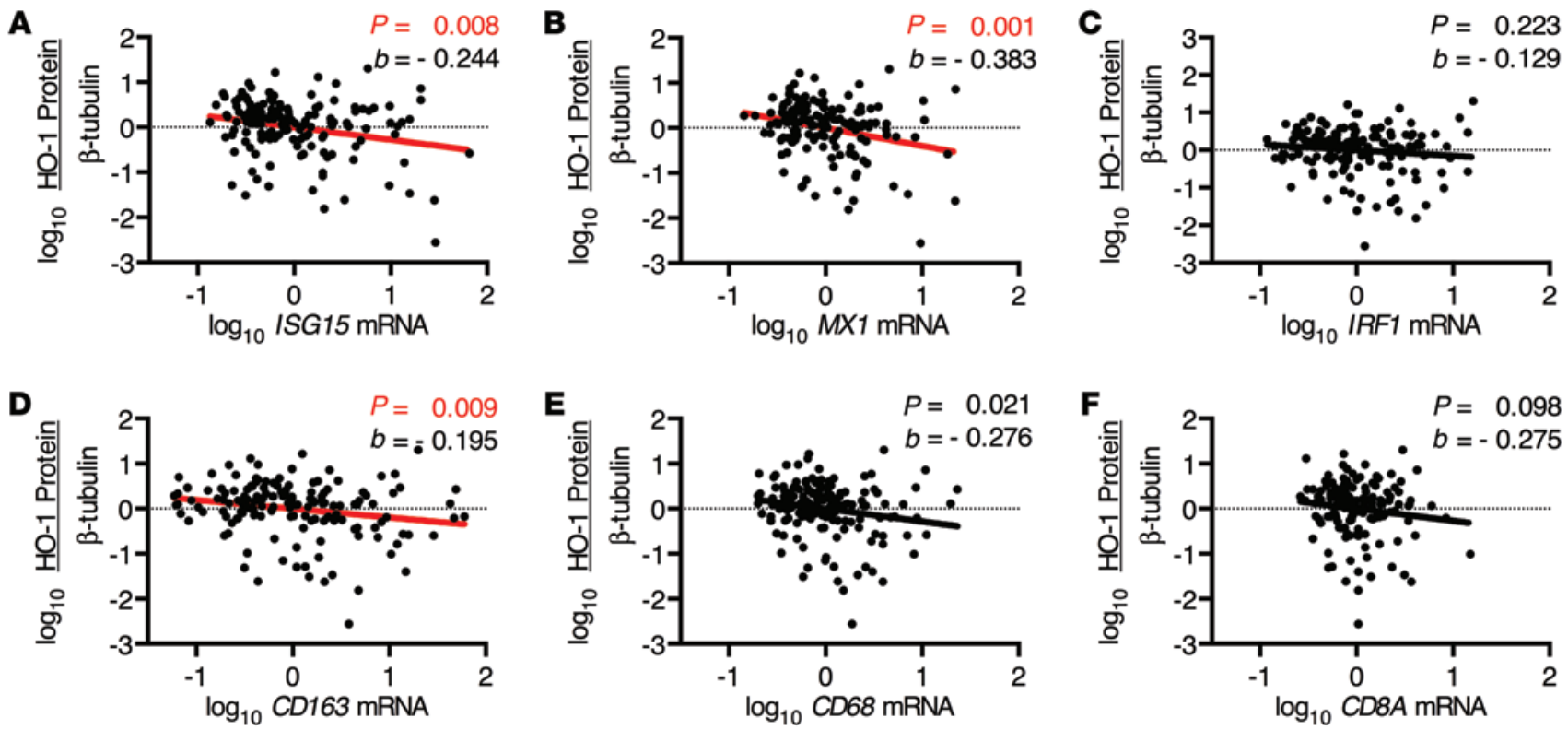

Figure 4. DLPFC HO-1 protein expression correlates with brain innate immune responses and macrophage markers. Expression of mRNA within the DLPFC was determined by qPCR (see Methods). Correlations were determined between DLPFC HO-1 protein expression, as determined by Western blot and densitometry analysis normalized to $\beta$-tubulin, and (A) ISG15, (B) MX1, (C) IRF1, (D) CD163, (E) CD68, and (F) CD8A mRNA. Associations were determined by multivariate linear regression with $\alpha=0.01$. Red regression lines denote significant trends.

was associated with supernatant neurotoxicity as determined by loss of microtubule-associated protein 2 (MAP2) immunoreactivity in a cell-based ELISA of primary neurons exposed to HIV-MDM supernatants (Figure 5E). Consistent with previous reports, HIV infection of MDM did not induce MDM death (Figure 5F), indicating that MDM injury and associated membrane leakiness are unlikely to account for the elevated supernatant glutamate.

Pharmacologic inducers of $\mathrm{HO}-1$ expression and an inhibitor of $\mathrm{HO}$ enzymatic activity modulate HIV-MDM supernatant glutamate and neurotoxicity. Given the association between decreased HO-1 protein expression and glutamate production in HIV-MDM, we hypothesized that HO-1 modulates the release of glutamate from MDM and the associated neurotoxicity. To test this, we induced HO-1 expression in HIV-MDM with cobalt (III) protoporphyrin IX chloride (CoPP) and determined effects on supernatant glutamate and neurotoxicity (Figure 6). CoPP robustly induces HO-1 protein expression in MDM ( 20-fold) with limited induction of other ARE proteins (NQO1 and TRXR1, 1.5- to 2-fold) with no effect on HO-2, GPX1, or GSTP1 expression (Supplemental Figure 7, A and B). We applied CoPP to HIV-MDM during days 6 to 15 of HIV infection, when glutamate production and supernatant neurotoxicity are generally robust (see Figure 5). The addition of CoPP profoundly increased HO-1 expression in HIV-MDM while modestly increasing expression of NQO1 (Figure 6B). No effect on HIV replication was observed (Figure 6A). However, CoPP treatment significantly reduced supernatant glutamate (Figure 6, C and E) and associated neurotoxicity (Figure 6, D and F). A similar reduction in supernatant glutamate and neurotoxicity was observed as expected when viral replication was inhibited via treatment of HIV-MDM with the reverse-transcriptase (RT) inhibitor efavirenz. These data suggest that HO-1 induction may suppress HIV-mediated macrophage glutamate production and neurotoxicity independently of viral replication.
To further confirm the role for HO-1 in modulating macrophage glutamate production and neurotoxicity, we used tin(IV) mesoporphyrin IX dichloride (SnMP), a potent inhibitor of HO-1 and HO-2 enzymatic activity (44), which also induces HO-1 expression, through derepression of the HO-1 promoter (45). As expected, the addition of SnMP to HIV-MDM cultures under conditions identical to those of the CoPP treatments did not alter HIV replication and did induce HO-1 protein expression (Figure 7, A and B). Strikingly, SnMP treatment dose dependently increased supernatant glutamate (Figure 7, C and E) and neurotoxicity (Figure 7, D and F), suggesting that inhibition of heme oxygenase (HO-1 and/or HO-2) enzymatic activity enhances HIV-MDM glutamate production. Notably, SnMP also modestly increased NQO1 expression in MDM without altering HO-2, TRXR1, GSTP1, or GPX1 expression, similar to the effects of CoPP (Supplemental Figure 7, A and B). As both CoPP and SnMP have similar effects on ARE protein expression, but only SnMP inhibits HO-1 and HO-2 enzymatic activity, these data strongly suggest that heme oxygenase enzymatic activity mediates the effects on HIV-MDM glutamate production and neurotoxicity. Additionally, similar effects of SnMP were observed in uninfected MDM, with SnMP treatment dose dependently increasing supernatant glutamate and neurotoxicity (Supplemental Figure 8, A and B). Importantly, we did not observe SnMP cytotoxicity in MDM at the doses used (Supplemental Figure 8, C and D). This further supports the hypothesis that deficiency of heme oxygenase enzymatic activity in MDM may induce glutamate release and neurotoxicity.

Finally, inhibitors of NQO1 (dicoumarol, DCM) and GPX1 (mercaptosuccinic acid, MSA) enzymatic activity did not increase MDM supernatant glutamate or neurotoxicity (Supplemental Figure 8, A and B), further supporting the specificity of heme oxygenase effects. These results suggest that HO-1 modulates MDM glutamate production and neurotoxicity, and they further suggest 
A

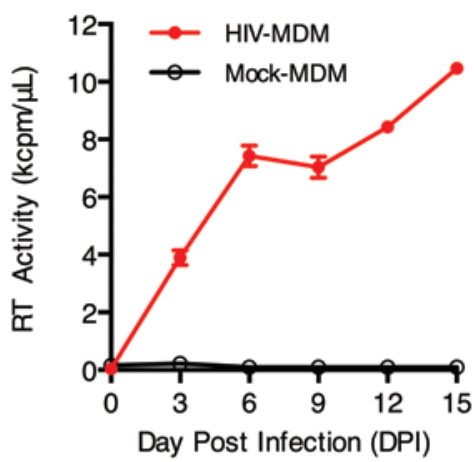

B

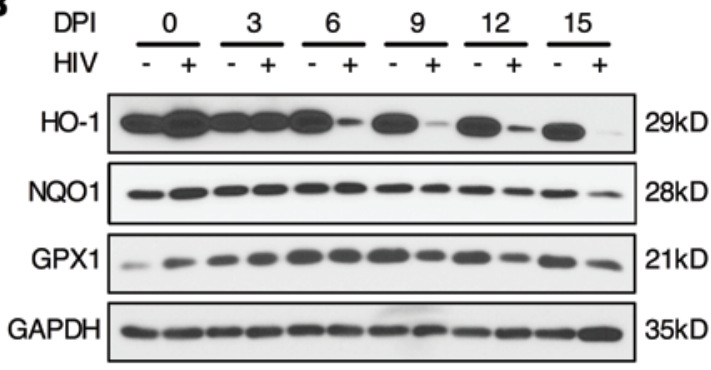

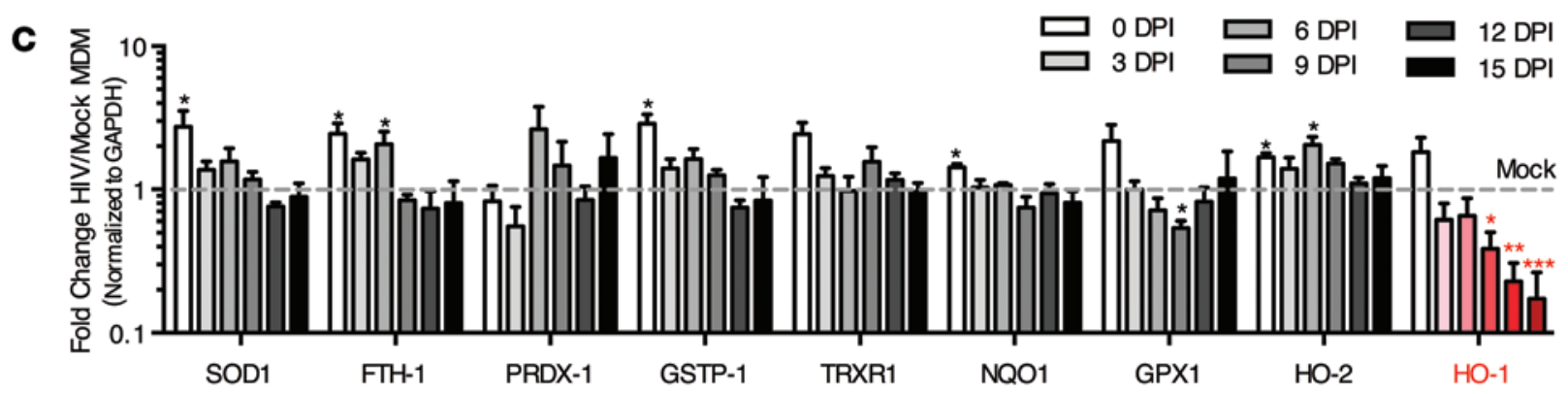

D

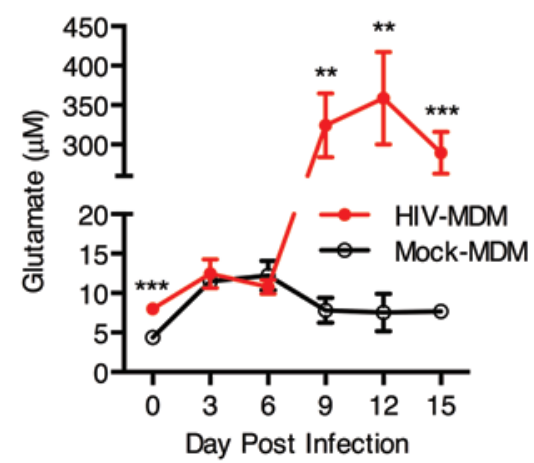

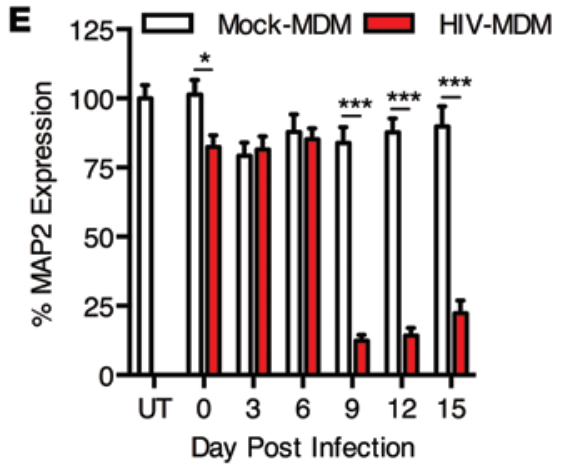

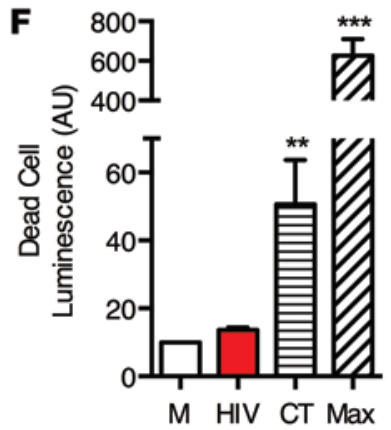

Figure 5. HIV infection of MDM reduces HO-1 protein expression and concomitantly increases supernatant glutamate levels and neurotoxicity. Infected (HIV-MDM) and mock-infected cultures (mock-MDM) were sampled and harvested every 3 days post infection (DPI) for analysis of supernatant glutamate content, supernatant neurotoxicity, and cellular protein expression. (A) Mean supernatant RT activity from representative HIV-infected cultures. (B) Western blot of selected ARE proteins (NQO1, HO-1, and GPX1) over the 15-day course of representative HIV-MDM infections. (C) Quantification of expression of canonical ARE proteins and HO-2 over the course of infection, as determined by Western blotting. Values represent mean \pm SEM ( $n=4$ different macrophage donor cultures) of the fold change in protein expression in HIV-infected versus mock-infected MDM. (D) Supernatant glutamate concentration and (E) supernatant neurotoxicity as measured by MAP2 expression in supernatant-exposed rat primary neuronal cultures) normalized to untreated (UT). RT, glutamate, and neurotoxicity data are representative of 4 independent experiments, with each replicate performed on MDM preparations from different donors. (F) Quantification of MDM cell death at 12 days after HIV infection. Results are averaged from 3 independent donors. Camptothecin (CT, 6 hour exposure) and complete cell lysis (max, maximum cytotoxicity) served as positive controls. All values represent mean \pm SEM. Statistical comparisons were made by paired Student's $t$ test. ${ }^{*} P<0.05 ;{ }^{* *} P<0.01 ;{ }^{* *} P<0.001$.

that the brain HO-1 deficiency observed in HIV-infected individuals directly contributes to HIV-mediated neurodegeneration.

siRNA-mediated induction and inhibition of HO-1 expression modulate HIV-MDM supernatant glutamate and neurotoxicity. Given our evidence that pharmacologic modulations of heme oxygenase alter supernatant glutamate and neurotoxicity, we attempted to confirm the role of HO-1 in HIV-MDM neurotoxin production using an siRNA-knockdown approach. We tested 2 siRNAs targeted against HO-1 and 2 siRNAs against $\underline{B}$ TB and $\underline{\mathrm{C} N C}$ homology $\underline{1}$ (BACH1) transcription factor, a robust negative transcriptional regulator of HO-1 (46). Transfection efficiency in 4 independent MDM donor cultures ranged from $84 \%$ to $96 \%$, as determined by transfection of a fluorescent control RNA oligomer (Supplemental Figure 9A). We confirmed that both siRNAs targeted against HO-1 reduced HO-1 protein expression by approximately 10-fold in MDM and HIVMDM, while siRNAs targeting BACH1 were able to reduce BACH1 protein expression by approximately 3-fold (Supplemental Figure 9, $\mathrm{B}-\mathrm{D})$. Associated with this BACH1 knockdown was an approximately 10-fold increase in HO-1 expression. Furthermore, neither the HO-1 nor BACH1 siRNAs altered HO-2 expression, in contrast with 2 siRNAs targeted to HO-2. Thus, we were able to selectively modulate HO-1 expression by siRNA without altering $\mathrm{HO}-2$ expression. 


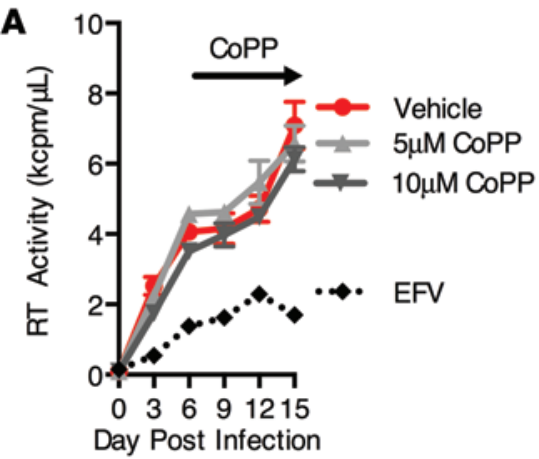

B
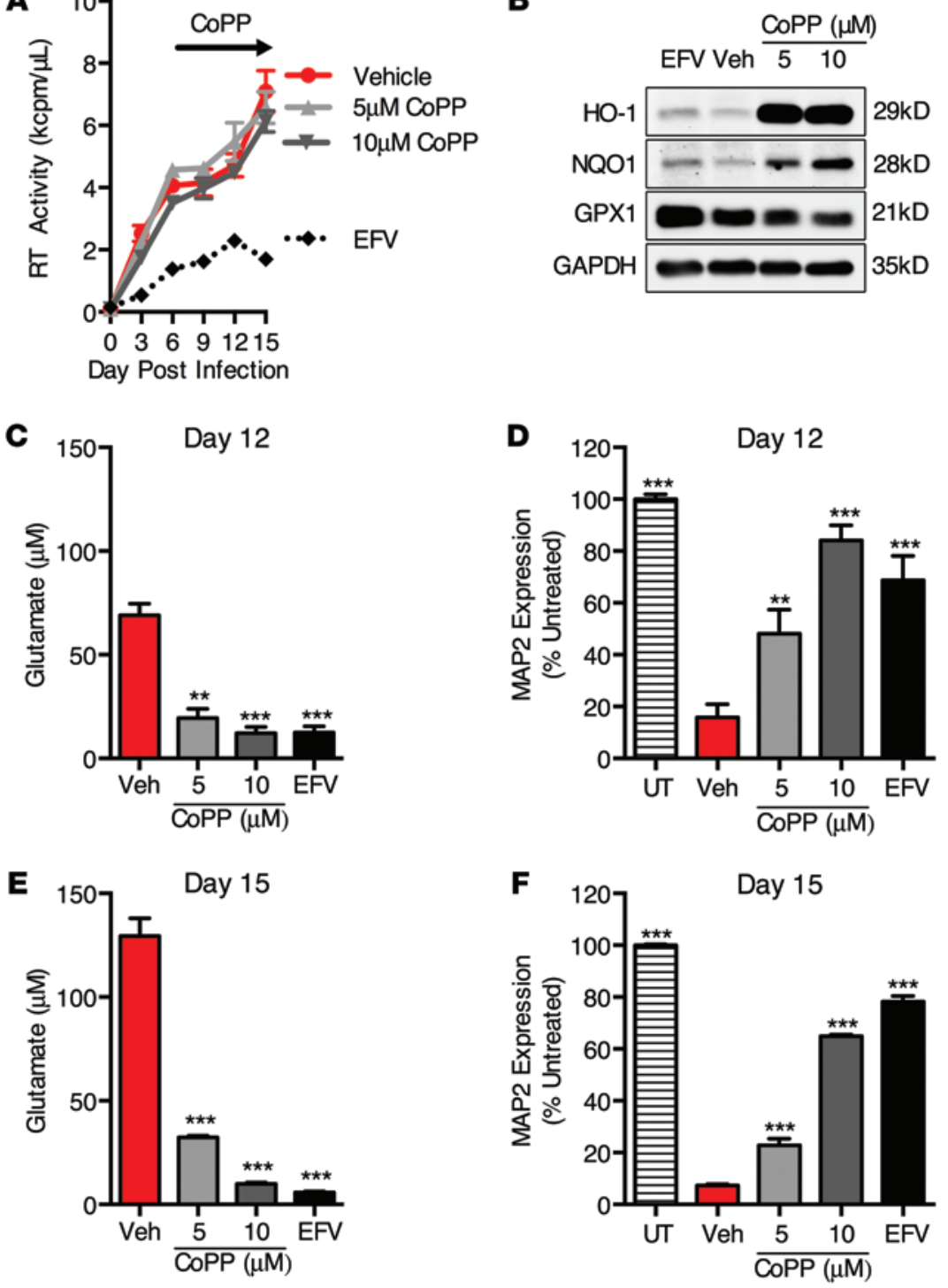

Figure 6. Inducers of H0-1 expression reduce HIV-MDM supernatant glutamate accumulation and neurotoxicity independently of HIV replication. CoPP, a potent inducer of HO-1, was added to HIV-MDM cultures on days 6, 9, and 12 post infection. Efavirenz (EFV) $(20 \mathrm{nM})$ was added 1 hour prior to HIV inoculation and replenished with each medium exchange to suppress HIV replication. (A) HIV replication (supernatant RT activity). (B) Western blot of cell lysates (day 12) for detection of ARE proteins HO-1, NQ01, and GPX1. (C) Supernatant (day 12) glutamate concentration and (D) supernatant neurotoxicity. (E) Supernatant (day 15) glutamate concentration and (F) supernatant neurotoxicity. Values represent mean $\pm \mathrm{SEM}$ of technical replicates from a representative experiment of 3 independent experiments, with each biological replicate performed on MDM preparations from a different donor. Statistical comparisons were made by 1-way ANOVA plus Hold-Sidak post hoc test. ${ }^{* *} P<0.01 ;{ }^{* * *} P<0.001$.

HO-1 and BACH1 double knockdown compared with BACH1 plus scramble, although HO-1 and BACH1 knockdown alone did increase and decrease neurotoxicity, respectively (not shown). Overall, these siRNA knockdown data support the conclusion that HO-1 modulates supernatant neurotoxicity and glutamate in HIV-MDM independently of HO-2.

The therapeutic ARE and HO-1 inducer, DMF, attenuates HIV-MDM supernatant glutamate and neurotoxicity. The potential benefit of therapeutic induction of HO-1 expression in the brains of HIVinfected individuals prompted us to examine the CNS-penetrating HO-1 inducer DMF for effects on MDM glutamate production and neurotoxicity. We previously showed that DMF and its primary in vivo metabolite, MMF, reduce neurotoxicity of HIVMDM supernatants when applied to MDM cultures prior to inoculation with HIV (37). This effect was associated with both increased HO-1 expression and reduced HIV replication, both of which likely

Therefore, to further confirm the role for HO-1 in regulating glutamate and neurotoxicity in HIV-MDM, we used siRNA targeting both HO-1 and BACH1 alone and in combination on day 6 after HIV infection of MDM. We transfected HIV-MDM with either scramble siRNA, HO-1 siRNA, BACH1 siRNA, BACH1 plus scramble siRNA, or BACH1 plus HO-1 siRNA and analyzed protein lysates and supernatants on day 12 after infection. Successful siRNA knockdown of HO-1 and BACH1 was confirmed by Western blot, and neither knockdown altered HIV replication (Figure 8, A and B). Knockdown with BACH1 siRNA (or BACH1 siRNA plus scramble siRNA) reduced extracellular glutamate and supernatant neurotoxicity, while knockdown of HO-1 increased extracellular glutamate and supernatant neurotoxicity (Figure 8, C and D). Furthermore, BACH1 plus HO-1 knockdown partially reversed the reduced glutamate and neurotoxicity effects, suggesting that BACH1 siRNA modulation of glutamate and neurotoxicity is in part an effect of $\mathrm{HO}-1$ protein induction (Figure 8, C and D). These siRNA-mediated effects on glutamate levels were seen in 2 additional independent experiments from 2 additional MDM donors. Among these 2 replicate experiments, one donor failed to achieve a significant increase in neurotoxicity with contributed to the reduction in neurotoxicity. We have now extended these studies to examine effects of DMF and MMF on glutamate production, HIV replication, and neurotoxicity when applied to MDM after HIV infection is established, as would reflect the state of the brain in HIV-infected individuals. Although less specific for HO-1 than CoPP, the ARE inducers DMF, MMF, and tert-butylhydroquinone (tBHQ), nonetheless significantly induce the expression of HO-1, as well as other ARE proteins (Supplemental Figure 10A). After exposure of MDM to each ARE inducer, we observed a peak of HO-1 induction at 6 to 12 hours, with HO-1 levels returning to baseline by 48 hours (Supplemental Figure 10, B-D).

Because of this transient effect on $\mathrm{HO}-1$ expression after single doses of DMF and MMF, we treated HIV-MDM cultures with multiple doses beginning on day 6 and again on day 9, and then quantified supernatant glutamate and neurotoxicity on day 12 (Figure 9). Similar to previous HO-1-modulating treatments, we saw no effect of either DMF or MMF on HIV replication (Figure 9, A and D), yet we observed a significant reduction in supernatant glutamate (Figure 9, B and E) and supernatant neurotoxicity (Figure 9, C and F). We saw similar effects after treatment with tBHQ (Figure 9, G-I). 

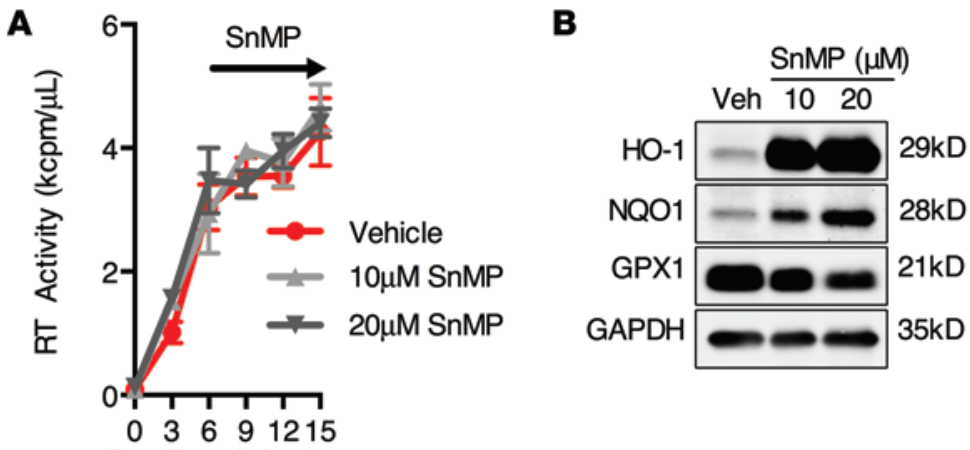

Day Post Infection
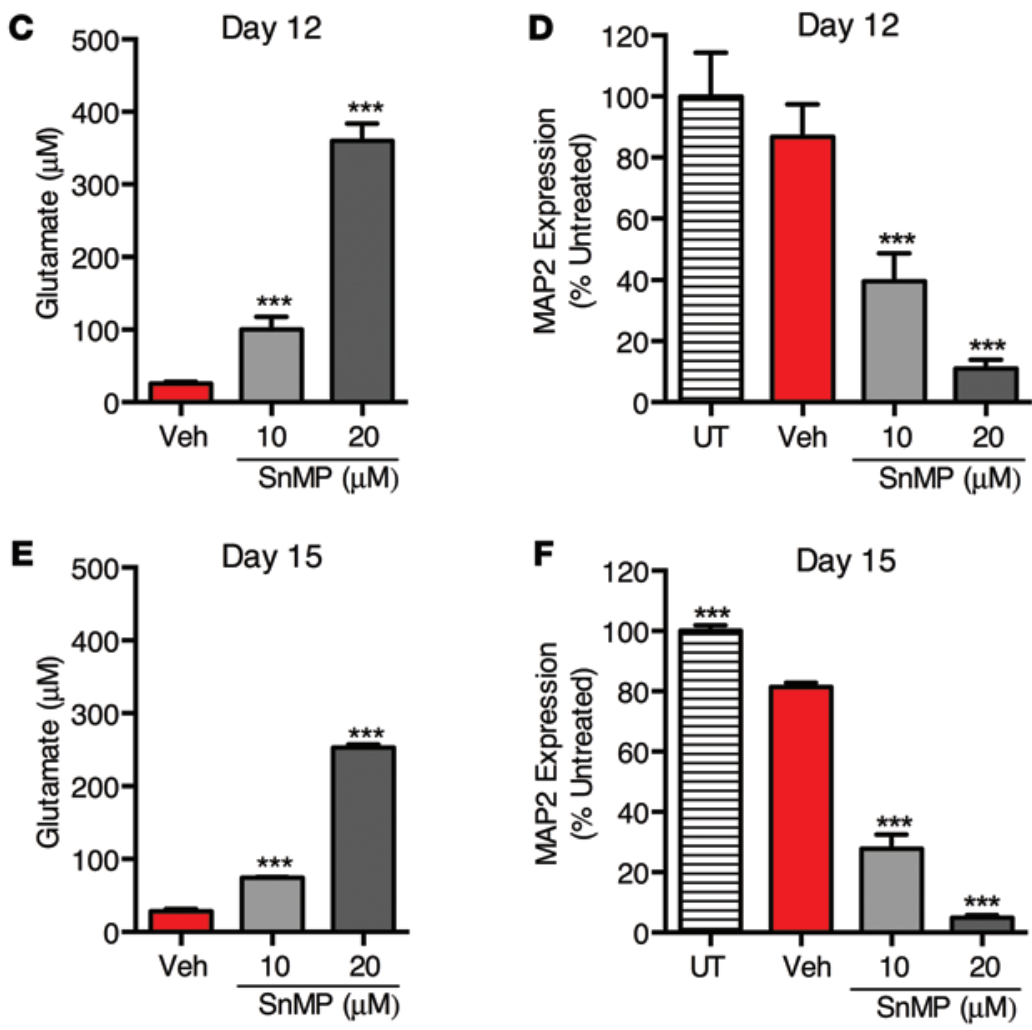

Figure 7. Exposure of HIV-MDM to an inhibitor of heme oxygenase enzymatic activity (SnMP) enhances glutamate release and supernatant neurotoxicity independently of HIV replication. SnMP, an inhibitor of heme oxygenase activity, was added to HIV-MDM cultures on days 6, 9 , and 12 post infection. (A) HIV replication (supernatant RT activity). (B) Western blot of cell lysates (day 15) for detection of ARE proteins HO-1, NQ01, and GPX1. (C) Supernatant (day 12) glutamate concentration and (D) supernatant neurotoxicity. (E) Supernatant (day 15) glutamate concentration and (F) supernatant neurotoxicity. Values represent mean \pm SEM of technical replicates from a representative experiment of 3 independent experiments, with each biological replicate performed on MDM preparations from a different donor. Statistical comparisons were made by 1-way ANOVA plus Hold-Sidak post hoc test. ${ }^{* *} P<0.001$.

HO-1 expression in conditions relevant to HIV CNS infection by Western blot. Such infection is associated with increased expression of systemic and CNS inflammatory mediators, including IFN- $\gamma$, TNF- $\alpha$, and lipopolysaccharide (LPS) (48-51). Initially we examined HO-1 protein expression in human fetal astrocytes following chronic exposure (15 days) to these inflammatory mediators to reflect the chronic inflammatory state in HIV-infected individuals. This chronic exposure to IFN- $\gamma$ alone or in combination with TNF- $\alpha$ or LPS significantly reduced HO-1 protein expression (Figure 10, A and B), while no effect of TNF- $\alpha$ or LPS on HO-1 expression was observed.

We next examined astrocyte $\mathrm{HO}-1$ expression after a 24-hour exposure to these inflammatory mediators. Under these conditions, TNF- $\alpha$ alone and in combination with LPS induced HO-1 expression (Figure 10, C and D), while IFN- $\gamma$ showed a nonsignificant decrease in HO-1 expression. Notably, this TNF- $\alpha$ induction of HO-1 was attenuated when IFN- $\gamma$ was applied in combination, suggesting that IFN- $\gamma$ can suppress $\mathrm{HO}-1$ induction by proinflammatory cytokines. We also found that IFN- $\gamma$ significantly reduced HO-1 expression in uninfected MDM after

Notably, treatment of primary rat cortical neurons with DMF or MMF concurrently with HIV-infected macrophage supernatant did not prevent neuronal injury and death as measured by MAP2 ELISA (Supplemental Figure 11, A and B), suggesting that DMF/ MMF neuroprotection in this study is likely due to effects on macrophages, rather than effects of any unmetabolized DMF/MMF remaining in the macrophage supernatant used to treat neurons. These data demonstrate that ARE inducers, including DMF and MMF, at concentrations consistent with CSF MMF concentrations in vivo (47) can ameliorate neurotoxin production in HIV-MDM independently of suppression of viral replication.

IFN- $\gamma$ reduces $\mathrm{HO}-1$ protein expression in human fetal astrocytes and uninfected macrophages. Because macrophages represent only a small fraction of cells within the CNS, reduction of HO-1 within HIV-infected macrophages alone is unlikely to account for the HO-1 deficiency we observed in whole-tissue lysates from the DLPFC and striatum. Because astrocytes are the predominant cell lineage expressing $\mathrm{HO}-1$ in the CNS, we examined astrocyte
24 hours of treatment (Supplemental Figure 12, A and B). These results suggest that the HO-1 deficiency in HIV-infected brain could result from macrophage HO-1 loss induced by HIV replication and astrocyte HO- 1 loss induced by IFN- $\gamma$ signaling. Consistent with this hypothesis, elevated CSF levels of IFN- $\gamma$ are found in $\mathrm{HIV}^{+}$patients, even those on suppressive ART (52).

\section{Discussion}

The high prevalence of HAND in ART-treated HIV-infected individuals strongly emphasizes the need for adjunctive therapies that target the neuropathological processes that persist within the CNS despite the substantial benefit provided by ART $(12,13)$. These processes include inflammation and oxidative stress, which persist not only within the CNS but also within the systemic compartment in such individuals (6-9). In our attempts to identify host factors that contribute to these processes, we have demonstrated a deficiency of the phase II detoxifying enzyme HO- 1 in the prefrontal cortex of HIV-infected individuals with HAND, and we 
A
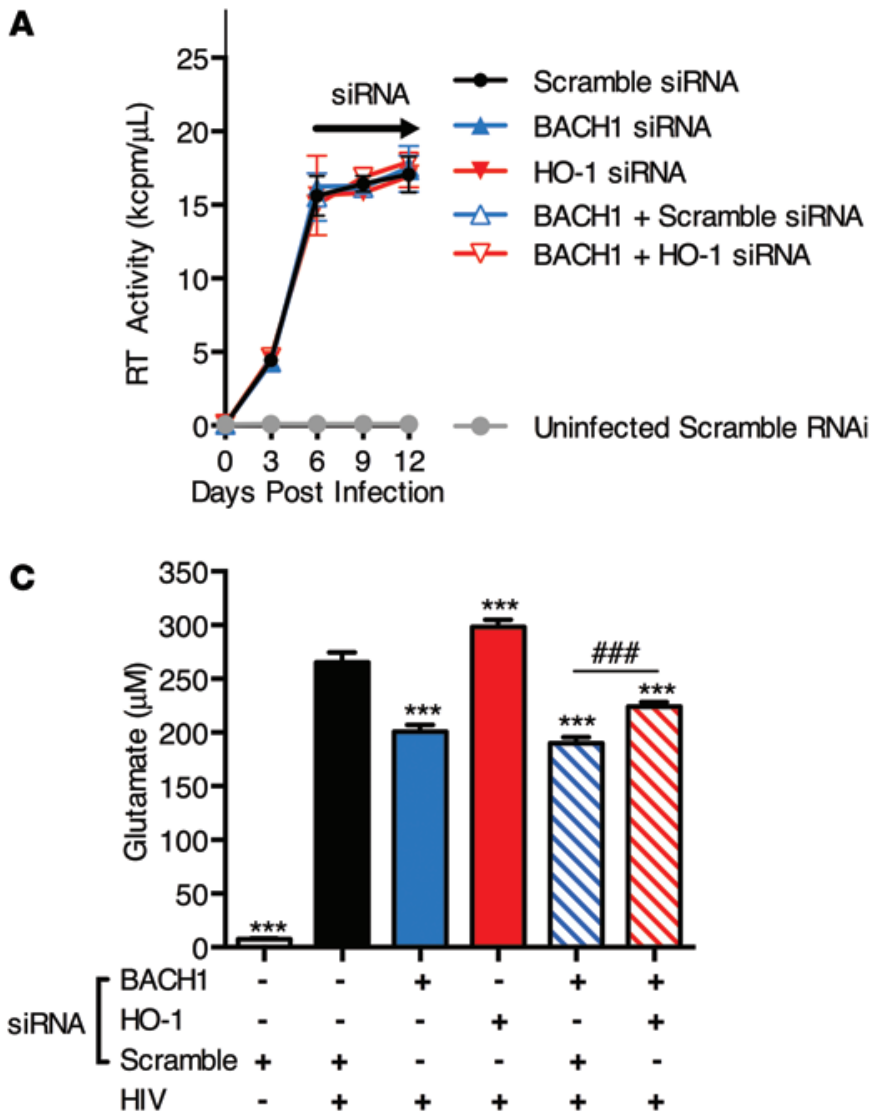

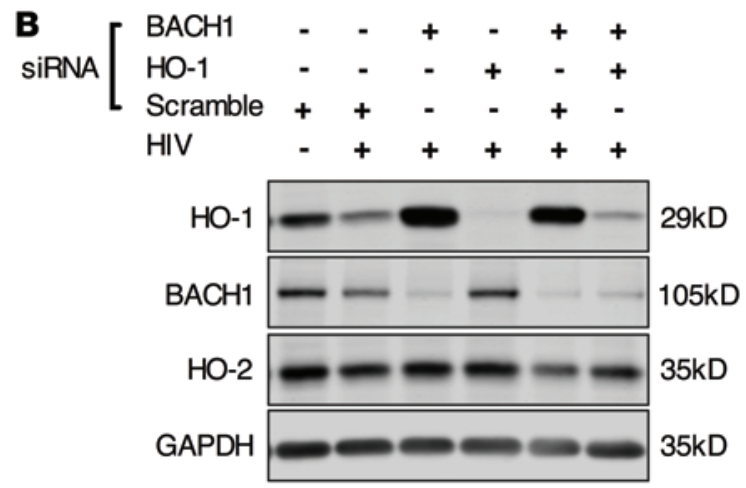

D

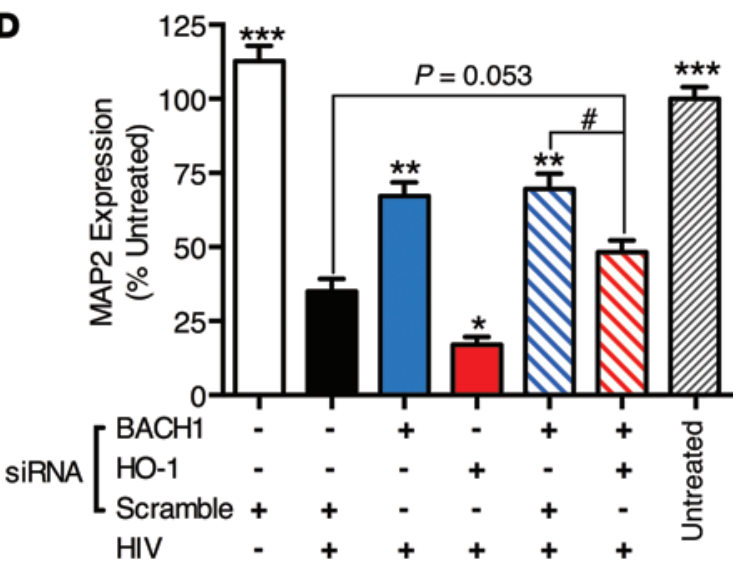

Figure 8. Knockdown of HO-1 and BACH1 by siRNA modulates extracellular glutamate and supernatant neurotoxicity in HIV-MDM independently of HIV replication. On day 6 post infection, siRNA targeting HO-1 or BACH1 or a scramble negative control siRNA were transfected into HIV-MDM alone or in combination using Lipofectamine RNAimax. On day 9, medium was fully exchanged without retransfection. Representative data from a single donor infection showing (A) HIV replication (supernatant RT activity), (B) Western blot of cell lysates (day 12) for detection of HO-1, BACH1, HO-2, and GAPDH, (C) supernatant (day 12) glutamate concentration, and (D) supernatant neurotoxicity. Values represent mean \pm SEM. Statistical comparisons were made by 1-way ANOVA plus Hold-Sidak post hoc test. ${ }^{*} P<0.05,{ }^{* *} P<0.01,{ }^{* *} P<0.001$ versus HIV-MDM scramble siRNA; ${ }^{*} P<0.05$, ${ }^{* \#} P<0.001$ for indicated comparison.

have further shown that this deficiency is particularly severe in those subjects who also have HIV encephalitis. This protein deficiency appears to be specific for HO-1 relative to other members of the ARE-driven gene family, as we saw no consistent changes in other ARE proteins or in the heme oxygenase isoform HO-2 in brain tissue. Prefrontal cortex HO-1 deficiency correlated with higher brain and CSF viral load and markers of immune activation and was associated with a clinical diagnosis of HAND. Notably, we observed no significant difference in the severity of brain HO-1 deficiency between ART-naive and ART-experienced subpopulations, suggesting that conventional ART may not prevent or reverse this brain HO-1 deficiency and its associated cognitive dysfunction. However, we cannot rule out an influence of ART, as ART-experienced decedents are often discontinued from therapy regimens near the time of death.

We have thus linked brain HO-1 deficiency to HIV replication, immune activation, and cognitive dysfunction (HAND) in ARTtreated and nontreated subjects. Furthermore, we have used our in vitro HIV neurotoxicity model to demonstrate that HIV infection of macrophages markedly reduces HO- 1 expression and that this HO-1 deficiency is linked to increased toxic levels of glutamate, a HAND-associated neurotoxin (41-43). This association between virus-induced HO-1 deficiency and glutamate production suggests a potential therapeutic benefit of restoring HO-1 expression in HIV-infected brain macrophages. To this end, we have shown that restoring HO-1 expression in HIV-infected macrophages in vitro reduces neurotoxic levels of glutamate. Because therapeutic formulations of drugs that can effectively induce HO-1 expression (e.g., the fumaric acid ester DMF) have become available clinically, our studies suggest a feasible and rational therapeutic approach to providing neuroprotection to HIV-infected patients.

In addition to demonstrating HO- 1 deficiency in the prefrontal cortex of $\mathrm{HIV}^{+}$patients with HAND, we also observed a reduction in $\mathrm{HO}-1$ protein expression in the striatum of subjects with HIVE compared with $\mathrm{HIV}^{-}$and $\mathrm{HIV}^{+} / \mathrm{HIVE}^{-}$subjects in a smaller cohort. This finding provides evidence that HIV-associated HO-1 deficiency within the CNS is not restricted to the prefrontal cortex, but occurs in other brain regions. However, we did not observe altered HO- 1 protein expression in the occipital or cerebellar cortex, indicating that the reduction of HO-1 in HIV infection is not a global HIV effect in the brain. The pathological mechanism underlying the regional distribution of HO-1 protein deficiency in HIV-infected individuals is unclear. Notably, structural and functional imaging studies as well as neuropsychologi- 

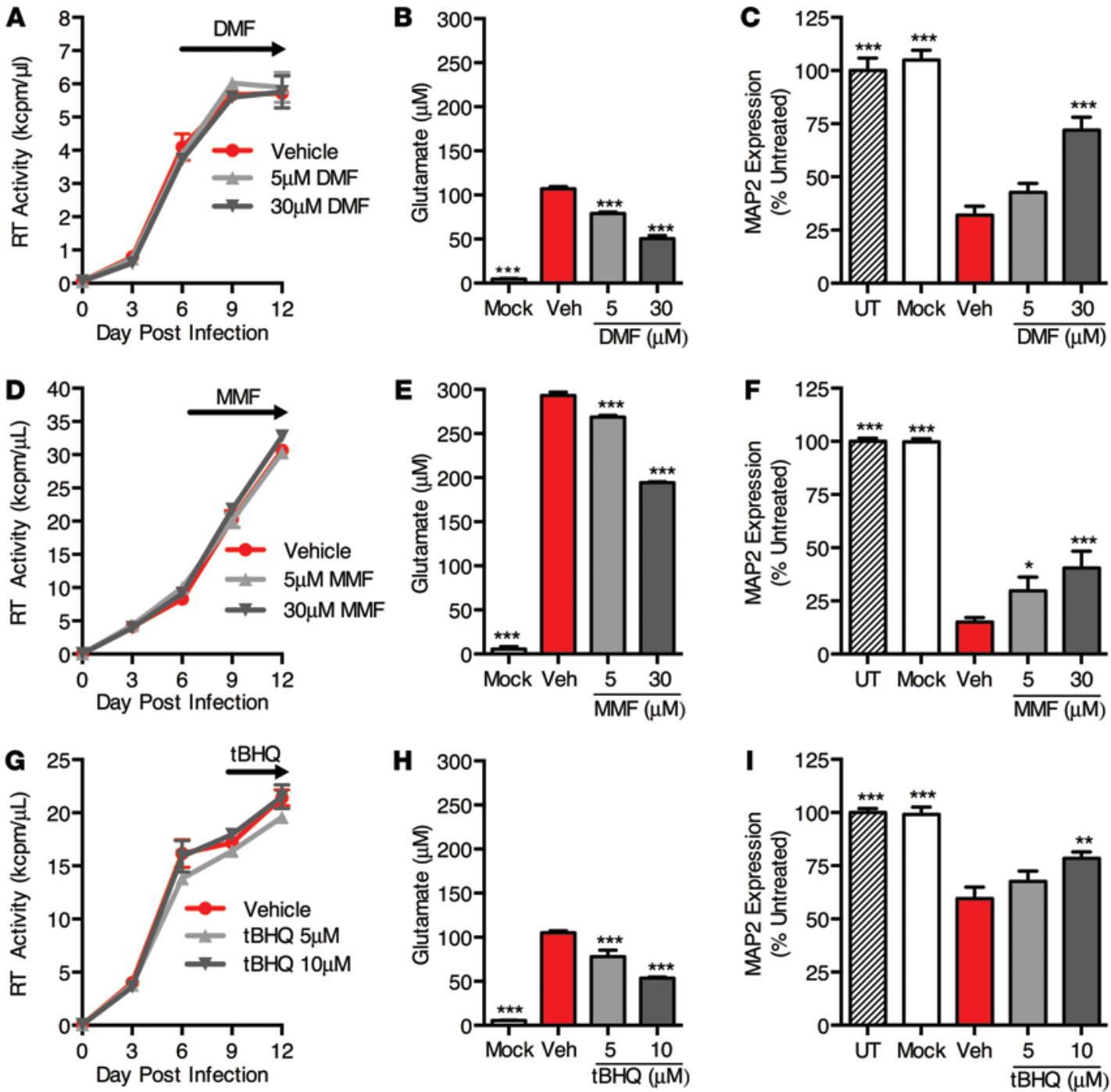

Figure 9. DMF and MMF reduce glutamate release and associated supernatant neurotoxicity in HIV-MDM independently of HIV replication. DMF and MMF were added to HIV-MDM on day 6 post infection and replenished on day 9 post infection. tBHQ was added on day 9 post infection and not replenished. Culture supernatants (day 12 post infection) were assayed for glutamate concentration and neurotoxicity normalized to untreated primary rat neuronal cultures (UT). (A) HIV replication (supernatant RT activity), (B) supernatant glutamate concentration, and (C) supernatant neurotoxicity in DMF-treated HIV-MDM. Similar effects of DMF treatment in HIV-MDM were observed in 4 of 5 independent experiments, with each replicate performed on MDM preparations from a different donor. (D) HIV replication (supernatant RT activity), (E) supernatant glutamate concentration, and (F) supernatant neurotoxicity in MMF-treated HIV-MDM. Similar effects of MMF treatment in HIV-MDM were observed in 3 of 4 independent experiments, with each replicate performed on MDM preparations from a different donor. (G) HIV replication (supernatant RT activity), (H) supernatant glutamate concentration, and (I) supernatant neurotoxicity in tBHQ-treated HIV-MDM. Similar effects of tBHQ treatment in HIV-MDM were observed in 3 of 3 independent experiments, with each replicate performed on MDM preparations from a different donor. Values represent mean \pm SEM. Statistical comparisons were made by 1-way ANOVA plus Hold-Sidak post hoc test. Veh, vehicle. ${ }^{*} P<0.05{ }^{* *} P<0.01 ;{ }^{* *} P<0.001$ versus vehicle.

cal testing studies suggest that the frontostriatal circuitry may be particularly vulnerable to injury and dysfunction in HIV infection (53). Further analysis of regional expression of immune activation markers, viral load, and other proteins may elucidate common or unique associations with HO-1 expression.

Previous studies have associated CNS HIV replication with neurodegeneration, immune activation, and cognitive dysfunction and have further shown that persistence of immune activation and oxidative stress in both systemic and CNS compartments is associated with disease progression within those compartments (6-9). We demonstrated that prefrontal cortex HO-1 deficiency in HIV-infected sub- jects correlates with increased brain parenchyma and CSF viral loads, but not with plasma viral load or CD4 T cell count. This implicates the CNS viral reservoir as a driver of brain HO-1 deficiency independently of systemic HIV reservoirs, and it could identify one mechanism, loss of HO-1 enzymatic function, that contributes to persistent oxidative stress in HIV-infected brain.

Whether HO-1 deficiency within the peripheral compartment is similarly associated with systemic oxidative stress and systemic HIV disease progression is unknown. However, a recent study by Seu et al. has shown that a GT(n) repeat polymorphism within the HO-1 promoter region is associated with 
A

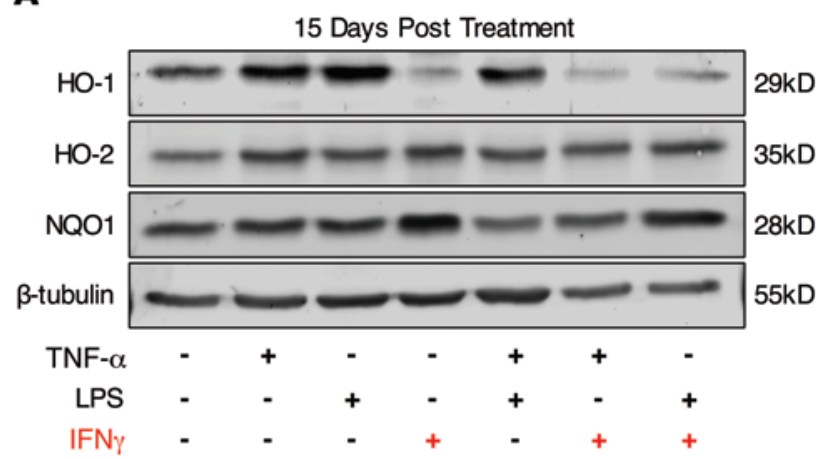

C

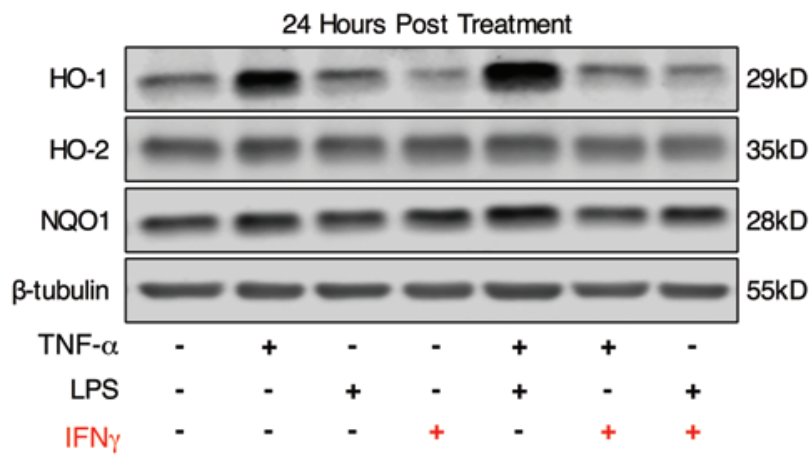

B

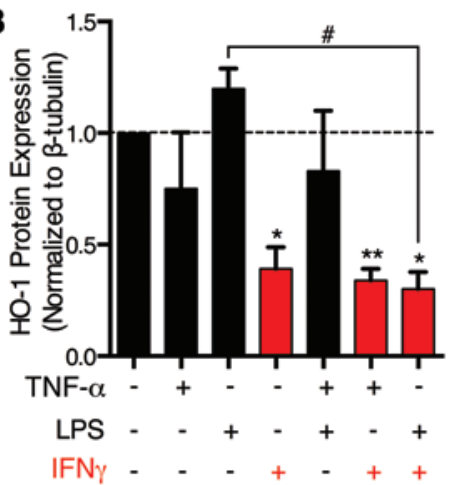

D

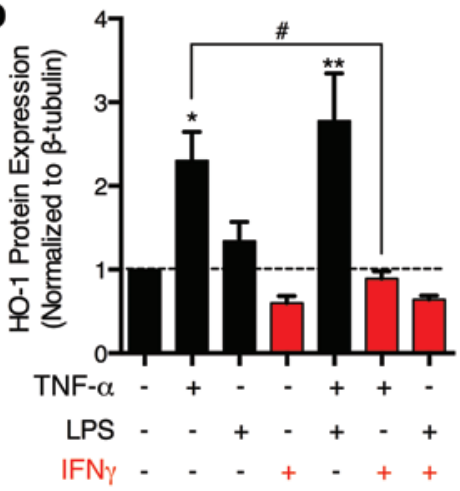

Figure 10. IFN- $\gamma$ reduces H0-1 protein expression in primary human fetal astrocytes. Human fetal astrocytes were treated with IFN- $\gamma(10 \mathrm{ng} / \mathrm{ml})$, TNF- $\alpha$ $(10 \mathrm{ng} / \mathrm{ml})$, and LPS $(1 \mu \mathrm{g} / \mathrm{ml})$ alone or in combination for 24 hours or 15 days. Medium was changed and immune modulators replaced every 3 days. Following treatment, cells were lysed and protein expression of HO-1, HO-2, NQO1, and $\beta$-tubulin was analyzed by Western blot. (A) Representative Western blot and (B) densitometry quantification of HO-1 expression normalized to $\beta$-tubulin in human fetal astrocytes following 15 days of exposure to immune modulators $(n=4)$. (C) Representative Western blot and (D) densitometry quantification of HO-1 expression normalized to $\beta$-tubulin in human fetal astrocytes following 24 hours of exposure to immune modulators $(n=3)$. Values represent mean \pm SEM. Statistical comparisons were made by 1 -way ANOVA plus Hold-Sidak post hoc test. ${ }^{*} P<0.05,{ }^{* *} P<0.01$ versus vehicle; ${ }^{\#} P<0.05$ for indicated comparison.

altered HO-1 inducibility in PBMCs. Specifically, longer GT(n) repeat length is associated with lower HO-1 induction. Furthermore, longer GT(n) repeat length in HIV-infected African Americans correlated with increased plasma sCD14 and viral load, suggesting a link with systemic HIV disease progression (54). Additional studies of the associations between HO-1 microsatellite polymorphisms and risk for HIV disease progression, particularly neurocognitive impairment, in larger population cohorts are clearly needed.

In addition to correlating with CNS viral replication, prefrontal cortex HO-1 deficiency also correlated with markers of innate immunity (type I IFN response genes ISGI5 and MX1) and macrophage and microglia activation (CD163). These data implicate the potential role for macrophage and microglia activation/infiltration in mediating brain HO-1 deficiency, although how this occurs is as yet unclear. We have demonstrated that HIV replication in macrophages dramatically reduces HO- 1 expression and that this HO-1 deficiency is associated with elevated extracellular glutamate and supernatant neurotoxicity.

Using both pharmacological and genetic approaches, we further showed that HO-1 induction decreased glutamate production and associated neurotoxicity. However, the contribution of other ARE-induced genes (e.g., NQO1), which were induced to varying extents by these approaches, cannot be ruled out. The inhibition of heme oxygenase (HO-1 and HO-2) enzymatic activity and selective HO-1 knockdown increased glutamate and associated neurotoxicity in HIV-infected macrophages, which further implicates HO-1 in modulating macrophage-mediated neurotoxin production in HIV infection. Given that microglia can support robust HIV replication and that HIV infection of microglia similarly enhances extracellular glutamate and associated neurotoxicity (42), we hypothesize that HIV reduces HO-1 expression and that HO-1 modulates neurotoxin production in HIV-infected microglia.

However, loss of HO-1 protein expression in macrophages/ microglia probably does not account for the low level of HO-1 expression in whole-brain tissue lysates. We speculate that HIV replication in macrophages/microglia indirectly modulates HO-1 expression in other cell types within the CNS, perhaps through release of cellular factors associated with macrophage/microglia activation and HIV-mediated neuroinflammation. Although HO-1 expression has been reported in neurons (although often undetectable in individuals without CNS disease), HO-1 appears to be primarily expressed in astrocytes, macrophages, and microglia in the normal/noninjured brain $(55,56)$. Because macrophages and microglia constitute a small percentage of the cells within the 
brain, even in HIV-encephalitis cases (57), most of the detectable HO-1 likely represents the astrocyte population, which accounts for approximately $50 \%$ of the brain parenchyma. We have identified IFN- $\gamma$ as a candidate cellular factor that decreases astrocyte HO-1 expression, which could thus be responsible for the HO-1 deficiency observed in the prefrontal cortex and striatum of HIVinfected individuals. IFN- $\gamma$ has been shown to be elevated in the CNS of HIV-infected individuals $(48,49)$, even aviremic subjects on suppressive ART (52). We have shown that IFN- $\gamma$ can reduce the expression of HO-1 in both human astrocytes and noninfected human macrophages, a finding previously described in IFN- $\gamma$ treated human glioblastoma cells and human retinal pigment endothelial cells $(58,59)$. Thus, elevated IFN- $\gamma$ within the CNS of HIV-infected individuals could mediate reduction of HO-1 expression in noninfected cells. Because macrophage glutamate production is strongly associated with HO-1 deficiency, we suspect a similar relationship between HO-1 deficiency and glutamate production in astrocytes, which are the primary scavengers of extracellular glutamate within the CNS.

We believe that the deficiency of HO-1 expression in HAND brain might be unique among neurodegenerative diseases associated with CNS inflammation and oxidative stress. Alzheimer disease, Parkinson disease, and multiple sclerosis are also associated with CNS inflammation and oxidative stress (60), yet are not associated with decreased brain HO-1 expression. In contrast, these neurodegenerative diseases are all associated with increased brain HO-1 (15-17). For such CNS diseases, HO-1 induction in response to injury is considered to be a limited endogenous protective response and a potential therapeutic target for further protection against inflammation and oxidative stress (4). Why HO-1 expression is reduced in HIV infection of the brain, which is also associated with inflammation and oxidative stress, is unclear; effects of IFN- $\gamma$ are one possibility. Our preliminary studies of HO-1 mRNA expression in HIV-infected brain and in HIV-infected macrophages do not indicate transcriptional suppression of HO-1 (not shown). Furthermore, other ARE-driven gene products, such as NQO1, GPX1 and others, are expressed at normal levels in HIV-infected brain and in HIV-infected macrophages, which argues against transcriptional suppression of the ARE promoter as an explanation for HO-1 deficiency. The deficiency of HO-1 expression in both systems appears relatively specific for $\mathrm{HO}-1$ among ARE proteins, and we suspect accelerated HO-1 degradation as the likely cause. We are currently investigating this hypothesis.

Our studies support a role for induction of HO-1 expression as a protective strategy against HIV disease progression, and they further suggest that drugs such as DMF could serve this therapeutic role. DMF and its primary in vivo metabolite MMF are potent inducers of $\mathrm{HO}-1$ expression and $\mathrm{MMF}$ is detectable within the CSF after oral delivery of DMF at concentrations $(4.4 \mu \mathrm{M})$ that effectively induce macrophage $\mathrm{HO}-1$ expression and suppress production of neurotoxic levels of glutamate (47). Other effects of DMF and MMF (inhibition of HIV infection of macrophages, inhibition of macrophage NF- $\kappa B$ nuclear translocation and TNF- $\alpha$ release, and reduction of CCL2-mediated monocyte chemotaxis) could also have beneficial effects in ART-treated individuals (37), although whether these effects are related to HO-1 induction is not clear.
Notably, HO-1 has been reported to inhibit NF- $\mathrm{BB}$ through inhibition of phosphorylation of the $\mathrm{p} 65$ (NF- $\mathrm{\kappa B}$ subunit) at serine 276 (61), a mechanism of inhibition also reported in response to DMF treatment in dendritic cells (62). Thus, inhibition of NF- $\kappa \mathrm{B}$ signaling may play a role in the DMF/HO-1-mediated decrease in HIVinfected macrophage neurotoxin production. Considerable evidence therefore supports the testing of HO-1 inducers such as DMF as adjunctive therapy for prevention of both systemic and CNS complications of HIV infection in ART-treated subjects (reviewed in ref. 26) and additional studies of the role of HO-1 in HIV disease progression are needed.

\section{Methods}

Supplemental Methods. Detailed descriptions of human cohort characteristics and all protocols used can be found in Supplemental Methods and Supplemental Tables 1-6.

Statistics. All quantifications are expressed as mean \pm SEM. Twotailed Student's $t$ test or 1-way ANOVA followed by a Holm-Sidak post-test were performed on indicated comparisons. Analyses of linear trends were performed by multivariate linear regression analysis where $b$ is the slope of the line of association and $P$ is the $P$ value versus the null hypothesis $(b=0)$. Significance was defined as $\alpha=0.05$ unless otherwise noted in order to correct for multiple comparisons. Statistical support was provided by the Biostatics and Data Management Core, Center for AIDS Research, Perelman School of Medicine, University of Pennsylvania.

Study approval. All animal studies and protocols were carried out in accordance with the NIH Guide for the Care and Use of Laboratory Animals (8th edition. Revised 2011) and approved by the University of Pennsylvania IACUC. All human studies and protocols for monocyte isolation were reviewed and approved by the University of Pennsylvania IRB, and all participants provided written informed consent. Isolation of human fetal astrocytes from fetal brain tissue was performed in full compliance with NIH ethical guidelines and approved by the IRB of Temple University. All NNTC studies were conducted in accordance with human subject protection protocols at participating institutions. Written consent was obtained for subjects at 4 collection sites in the USA. The following offices maintained the IRBs that provided oversight for the protection of human subjects: (a) The University of Texas Medical Branch Office of Research Subject Protections, Galveston, Texas, USA; (b) Mount Sinai Medical Center Program for the Protection of Human Subjects, New York, New York, USA; (c) University of California, San Diego, Human Research Protections Program, San Diego, California, USA; (d) University of California, Los Angeles, Office of the Human Research Protection Program, Los Angeles, California, USA.

\section{Acknowledgments}

We acknowledge the support of the Center for AIDS Research Virology Core and Biostatistics and Data Management Core and the Mahoney Institute of Neurological Sciences (MINS) Neuron Culture Service Center at the University of Pennsylvania Perelman School of Medicine. Primary human fetal astrocytes were generously provided by Temple University's Comprehensive NeuroAIDS Center (P30 MH092177). This work was supported in part by NIH R01 grants NSO43994 (to D.L. Kolson), MH095671 (to D.L. Kolson), MH104134 (to D.L. Kolson), and 
NS072005 (to B.B. Gelman); T32 grants AI007632 (to A.J. Gill) and AG000255 (to S.A. Cross); and F30 grant MH102120 (to A.J. Gill). The NNTC provided tissue specimens and data and received support from MH083507, MH083501, MH083500, MH083506, and MH083545.
Address correspondence to: Dennis L. Kolson, Department of Neurology, Perelman School of Medicine, University of Pennsylvania, 415 Curie Boulevard, 280C Clinical Research Building, Philadelphia, Pennsylvania, USA. Phone: 215.573.3505; E-mail: kolsond@mail.med.upenn.edu.
1. Keyse SM, Tyrrell RM. Heme oxygenase is the major 32-kDa stress protein induced in human skin fibroblasts by UVA radiation, hydrogen peroxide, and sodium arsenite. Proc Natl Acad Sci US A. 1989;86(1):99-103.

2. Camhi SL, Alam J, Otterbein L, Sylvester SL, Choi AM. Induction of heme oxygenase-1 gene expression by lipopolysaccharide is mediated by AP-1 activation. Am J Respir Cell Mol Biol. 1995;13(4):387-398.

3. Rizzardini M, Terao M, Falciani F, Cantoni L. Cytokine induction of haem oxygenase mRNA in mouse liver. Interleukin 1 transcriptionally activates the haem oxygenase gene. Biochem J. 1993;290(1):343-347.

4. Cuadrado A, Rojo AI. Heme oxygenase-1 as a therapeutic target in neurodegenerative diseases and brain infections. Curr Pharm Des. 2008;14(5):429-442.

5. Ambegaokar SS, Kolson DL. Heme oxygenase-1 dysregulation in the brain: implications for HIVassociated neurocognitive disorders. Curr HIV Res. 2014;12(3):174-188.

6. Roc AC, et al. Detection of human immunodeficiency virus induced inflammation and oxidative stress in lenticular nuclei with magnetic resonance spectroscopy despite antiretroviral therapy. Arch Neurol. 2007;64(9):1249-1257.

7. Eden A, Price RW, Spudich S, Fuchs D, Hagberg $\mathrm{L}$, Gisslen M. Immune activation of the central nervous system is still present after $>4$ years of effective highly active antiretroviral therapy. J Infect Dis. 2007;196(12):1779-1783.

8. Ryan LA, et al. Plasma levels of soluble CD14 and tumor necrosis factor- $\alpha$ type II receptor correlate with cognitive dysfunction during human immunodeficiency virus type 1 infection. J Infect Dis. 2001;184(6):699-706.

9. Bandaru VV, et al. Associative and predictive biomarkers of dementia in HIV-1-infected patients. Neurology. 2007;68(18):1481-1487.

10. Kraft-Terry SD, Buch SJ, Fox HS, Gendelman HE. A coat of many colors: neuroimmune crosstalk in human immunodeficiency virus infection. Neuron. 2009;64(1):133-145.

11. Antinori A, et al. Updated research nosology for HIV-associated neurocognitive disorders. Neurology. 2007;69(18):1789-1799.

12. Simioni S, et al. Cognitive dysfunction in HIV patients despite long-standing suppression of viremia. AIDS. 2010;24(9):1243-1250.

13. Heaton RK, et al. HIV-associated neurocognitive disorders persist in the era of potent antiretroviral therapy: CHARTER Study. Neurology. 2010;75(23):2087-2096.

14. Gozzelino R, Jeney V, Soares MP. Mechanisms of cell protection by heme oxygenase-1. Annu Rev Pharmacol Toxicol. 2010;50:323-354.

15. Schipper HM, Liberman A, Stopa EG. Neural heme oxygenase- 1 expression in idiopathic Par- kinson's disease. Exp Neurol. 1998;150(1):60-68.

16. Schipper HM, Cisse S, Stopa EG. Expression of heme oxygenase- 1 in the senescent and Alzheimer-diseased brain. Ann Neurol. 1995;37(6):758-768.

17. Mehindate K, et al. Proinflammatory cytokines promote glial heme oxygenase-1 expression and mitochondrial iron deposition: implications for multiple sclerosis. J Neurochem. 2001;77(5):1386-1395.

18. Lin $\mathrm{Q}$, et al. Heme oxygenase-1 protein localizes to the nucleus and activates transcription factors important in oxidative stress. J Biol Chem. 2007;282(28):20621-20633.

19. Hori R, et al. Gene transfection of H25A mutant heme oxygenase- 1 protects cells against hydroperoxide-induced cytotoxicity. J Biol Chem. 2002;277(12):10712-10718.

20. Ishii T, et al. Transcription factor Nrf2 coordinately regulates a group of oxidative stressinducible genes in macrophages. J Biol Chem. 2000;275(21):16023-16029.

21. Yet SF, et al. Cardiac-specific expression of heme oxygenase- 1 protects against ischemia and reperfusion injury in transgenic mice. Circ Res. 2001;89(2):168-173.

22. Minamino T, et al. Targeted expression of heme oxygenase-1 prevents the pulmonary inflammatory and vascular responses to hypoxia. Proc Natl Acad Sci U S A. 2001;98(15):8798-8803.

23. Otterbein LE, Kolls JK, Mantell LL, Cook JL, Alam J, Choi AM. Exogenous administration of heme oxygenase- 1 by gene transfer provides protection against hyperoxia-induced lung injury. J Clin Invest. 1999;103(7):1047-1054.

24. Panahian N, Yoshiura M, Maines MD. Overexpression of heme oxygenase- 1 is neuroprotective in a model of permanent middle cerebral artery occlusion in transgenic mice. J Neurochem. 1999;72(3):1187-1203.

25. Ferrandiz ML, Devesa I. Inducers of heme oxygenase-1. Curr Pharm Des. 2008;14(5):473-486.

26. Gill AJ, Kolson DL. Dimethyl fumarate modulation of immune and antioxidant responses: application to HIV therapy. Crit Rev Immunol. 2013;33(4):307-359.

27. O'Donnell LA, Agrawal A, Jordan-Sciutto KL, Dichter MA, Lynch DR, Kolson DL. Human immunodeficiency virus (HIV)-induced neurotoxicity: roles for the NMDA receptor subtypes. JNeurosci. 2006;26(3):981-990.

28. Cook DR, et al. NMDA receptor modulation by the neuropeptide apelin: implications for excitotoxic injury. J Neurochem. 2011;118(6):1113-1123.

29. Nguyen TP, Soukup VM, Gelman BB. Persistent hijacking of brain proteasomes in HIV-associated dementia. Am J Pathol. 2010;176(2):893-902.

30. Gelman BB, et al. Neurovirological correlation with HIV-associated neurocognitive disorders and encephalitis in a HAART-era cohort. J Acquir Immune Defic Syndr. 2013;62(5):487-495.

31. Gelman BB, et al. The National NeuroAIDS
Tissue Consortium brain gene array: two types of HIV-associated neurocognitive impairment. PLoS One. 2012;7(9):e46178.

32. Horiuchi M, Itoh A, Pleasure D, Ozato K, Itoh T. Cooperative contributions of interferon regulatory factor 1 (IRF1) and IRF8 to interferongamma-mediated cytotoxic effects on oligodendroglial progenitor cells. J Neuroinflammation. 2011;8(8):1-16.

33. Schaer DJ, et al. CD163 is the macrophage scavenger receptor for native and chemically modified hemoglobins in the absence of haptoglobin. Blood. 2006;107(1):373-380.

34. Roberts ES, Masliah E, Fox HS. CD163 identifies a unique population of ramified microglia in HIV encephalitis (HIVE). J Neuropathol Exp Neurol. 2004;63(12):1255-1264.

35. Kim WK, et al. CD163 identifies perivascular macrophages in normal and viral encephalitic brains and potential precursors to perivascular macrophages in blood. Am J Pathol. 2006;168(3):822-834.

36. Chen W, Sulcove J, Frank I, Jaffer S, Ozdener H, Kolson DL. Development of a human neuronal cell model for human immunodeficiency virus (HIV)-infected macrophage-induced neurotoxicity: apoptosis induced by HIV type 1 primary isolates and evidence for involvement of the Bcl-2/ Bcl-xL-sensitive intrinsic apoptosis pathway. J Virol. 2002;76(18):9407-9419.

37. Cross SA, et al. Dimethyl fumarate, an immune modulator and inducer of the antioxidant response, suppresses HIV replication and macrophage-mediated neurotoxicity: a novel candidate for HIV neuroprotection. JImmunol. 2011;187(10):5015-5025.

38. Giulian D, Vaca K, Noonan CA. Secretion of neurotoxins by mononuclear phagocytes infected with HIV-1. Science. 1990;250(4987):1593-1596.

39. Burdo TH, et al. Increased monocyte turnover from bone marrow correlates with severity of SIV encephalitis and CD163 levels in plasma. PLOS Pathog. 2010;6(4):e1000842.

40. Lyons JL, et al. Plasma SCD14 is a biomarker associated with impaired neurocognitive test performance in attention and learning domains in HIV infection. J Acquir Immune Defic Syndr. 2011;57(5):371-379.

41. Jiang ZG, et al. Glutamate is a mediator of neurotoxicity in secretions of activated HIV-1-infected macrophages. J Neuroimmunol. 2001;117(1-2):97-107.

42. Huang Y, et al. Glutaminase dysregulation in HIV-1-infected human microglia mediates neurotoxicity: relevant to HIV-1-associated neurocognitive disorders. J Neurosci. 2011;31(42):15195-15204

43. Ferrarese $\mathrm{C}$, et al. Increased glutamate in CSF and plasma of patients with HIV dementia. Neurology. 2001;57(4):671-675.

44. Drummond GS, Galbraith RA, Sardana MK, Kap- 
pas A. Reduction of the $\mathrm{C} 2$ and $\mathrm{C} 4$ vinyl groups of Sn-protoporphyrin to form Sn-mesoporphyrin markedly enhances the ability of the metalloporphyrin to inhibit in vivo heme catabolism. Arch Biochem Biophys. 1987;255(1):64-74.

45. Abate A, Zhao H, Wong RJ, Stevenson DK. The role of Bach1 in the induction of heme oxygenase by tin mesoporphyrin. Biochem Biophys Res Commun. 2007;354(3):757-763.

46. Sun J, et al. Hemoprotein Bach1 regulates enhancer availability of heme oxygenase-1 gene. ЕМВО J. 2002;21(19):5216-5224.

47. Linker RA, et al. Fumaric acid esters exert neuroprotective effects in neuroinflammation via activation of the Nrf2 antioxidant pathway. Brain. 2011;134(3):678-692.

48. Shapshak P, Duncan R, Minagar A, Rodriguez de la Vega P, Stewart RV, Goodkin K. Elevated expression of IFN-gamma in the HIV-1 infected brain. Front Biosci. 2004;9:1073-1081.

49. Griffin DE, McArthur JC, Cornblath DR. Neopterin and interferon-gamma in serum and cerebrospinal fluid of patients with HIV-associated neurologic disease. Neurology. 1991;41(1):69-74.

50. Vassallo M, et al. Relevance of lipopolysaccharide levels in HIV-associated neurocognitive impairment: the Neuradapt study. J Neurovirol. 2013;19(4):376-382.
51. Achim CL, Heyes MP, Wiley CA. Quantitation of human immunodeficiency virus, immune activation factors, and quinolinic acid in AIDS brains. JClin Invest. 1993;91(6):2769-2775.

52. Kamat A, et al. Monocyte activation markers in cerebrospinal fluid associated with impaired neurocognitive testing in advanced HIV infection. J Acquir Immune Defic Syndr. 2012;60(3):234-243.

53. Melrose RJ, Tinaz S, Castelo JM, Courtney MG, Stern CE. Compromised fronto-striatal functioning in HIV: an fMRI investigation of semantic event sequencing. Behav Brain Res. 2008;188(2):337-347.

54. Seu L, Burt TD, Witte JS, Martin JN, Deeks SG, McCune JM. Variations in the heme oxygenase-1 microsatellite polymorphism are associated with plasma CD14 and viral load in HIV-infected African-Americans. Genes Immun. 2012;13(3):258-267.

55. Hirose W, Ikematsu K, Tsuda R. Age-associated increases in heme oxygenase- 1 and ferritin immunoreactivity in the autopsied brain. Leg Med (Tokyo). 2003;5(suppl 1):S360-S366.

56. Schipper HM. Heme oxygenase expression in human central nervous system disorders. Free Radic Biol Med. 2004;37(12):1995-2011.

57. Fischer-Smith T, et al. Macrophage/microglial accumulation and proliferating cell nuclear anti- gen expression in the central nervous system in human immunodeficiency virus encephalopathy. Am J Pathol. 2004;164(6):2089-2099.

58. Takahashi K, Nakayama M, Takeda K, Fujia $\mathrm{H}$, Shibahara S. Suppression of heme oxygenase-1 mRNA expression by interferon-gamma in human glioblastoma cells. J Neurochem. 1999;72(6):2356-2361.

59. Udono-Fujimori R, et al. Expression of heme oxygenase- 1 is repressed by interferongamma and induced by hypoxia in human retinal pigment epithelial cells. Eur J Biochem. 2004;271(14):3076-3084.

60. Reynolds A, Laurie C, Mosley RL, Gendelman HE. Oxidative stress and the pathogenesis of neurodegenerative disorders. Int Rev Neurobiol. 2007;82:297-325.

61. Seldon MP, et al. Heme oxygenase-1 inhibits the expression of adhesion molecules associated with endothelial cell activation via inhibition of NF-kappaB RelA phosphorylation at serine 276. JImmunol. 2007;179(11):7840-7851.

62. Peng H, et al. Dimethyl fumarate inhibits dendritic cell maturation via nuclear factor kappaB (NF-kappaB) and extracellular signal-regulated kinase 1 and 2 (ERK1/2) and mitogen stressactivated kinase 1 (MSK1) signaling. J Biol Chem. 2012;287(33):28017-28026. 